\title{
Review Article \\ Primary CNS Lymphomas: Challenges in Diagnosis and Monitoring
}

\author{
C. Chiavazza $\left(\mathbb{D},{ }^{1}\right.$ A. Pellerino, ${ }^{1}$ F. Ferrio, ${ }^{2}$ A. Cistaro $\left(\mathbb{D},{ }^{3}\right.$ R. Soffietti, ${ }^{1}$ and R. Rudà ${ }^{1}$ \\ ${ }^{1}$ Department of Neuro-Oncology, University \& City of Health \& Science Hospital, Turin, Italy \\ ${ }^{2}$ Department of Neuro-Radiology, University \& City of Health \& Science Hospital, Turin, Italy \\ ${ }^{3}$ Positron Emission Tomography Center IRMET, Turin, Italy \\ Correspondence should be addressed to C. Chiavazza; lottach@libero.it
}

Received 30 October 2017; Revised 12 April 2018; Accepted 17 May 2018; Published 21 June 2018

Academic Editor: Pasquale De Bonis

Copyright ( 92018 C. Chiavazza et al. This is an open access article distributed under the Creative Commons Attribution License, which permits unrestricted use, distribution, and reproduction in any medium, provided the original work is properly cited.

\begin{abstract}
Primary Central Nervous System Lymphoma (PCNSL) is a rare neoplasm that can involve brain, eye, leptomeninges, and rarely spinal cord. PCNSL lesions most typically enhance homogeneously on T1-weighted magnetic resonance imaging (MRI) and appear T2-hypointense, but high variability in MRI features is commonly encountered. Neurological symptoms and MRI findings may mimic high grade gliomas (HGGs), tumefactive demyelinating lesions (TDLs), or infectious and granulomatous diseases. Advanced MRI techniques (MR diffusion, spectroscopy, and perfusion) and metabolic imaging, such as Fluorodeoxyglucose Positron Emission Tomography (FDG-PET) or amino acid PET (usually employing methionine), may be useful in distinguishing these different entities and monitoring the disease course. Moreover, emerging data suggest a role for cerebrospinal fluid (CSF) markers in predicting prognosis and response to treatments. In this review, we will address the challenges in PCNSL diagnosis, assessment of response to treatments, and evaluation of potential neurotoxicity related to chemotherapy and radiotherapy.
\end{abstract}

\section{Introduction}

Primary Central Nervous System Lymphoma (PCNSL) is a rare extranodal non-Hodgkin lymphoma (NHL), which can involve brain, eye leptomeninges, and rarely spinal cord without evidence of systemic disease. PCNSL accounts for 1 to $3 \%$ of all NHL and about $3 \%$ of all primary brain tumours [1]. Approximately 95\% of PCNSLs are diffuse large B-cell lymphomas, while the other 5\% include T-cell, Burkitt, lymphoblastic, and marginal zone lymphomas [2]. Median age at disease presentation is 65 years, with a trend toward an increase of incidence in the oldest population [1, 3]. Risk factors for PCNSL include congenital and acquired immunosuppression (particularly HIV and posttransplant conditions).

Several pathogenetic mechanisms have been related to PCNSL, namely, mutations in specific genes (e.g., MYD88, PIM1, ATM, and TP53) and dysregulation in JAK/STAT, NF$\mathrm{kB}$, toll-like receptor, and B-cell receptor signaling pathways. PCNSL frequently shows 9p.24/PD-L1/PD-2 translocations and copy number alterations compared to other large B-cell lymphomas. Moreover, PCNSL has a selective angiotropism: the accumulation of tumor cells around blood vessels is thought to be the main cause of disruption of blood-brain barrier (BBB) [4]. From a pathological point of view, PCNSL is a "whole-brain disease" $[5,6]$, an important concept for both diagnosis and treatment.

In this review, we will discuss the challenges in the diagnosis and monitoring of PCNSL and also focus on baseline and long-term cognitive profile of PCSNL patients.

\section{Clinical Aspects}

PCNSL presentation is commonly subacute with typical symptoms such as cognitive decline or personality changes, confusion, focal neurological deficits, headache, and/or nausea and vomiting due to intracranial hypertension. Sometimes rapid worsening of neurological status up to stupor can be seen. Seizures are less frequent in comparison to other brain tumors (10\%-20\% of patients) [7]. Leptomeningeal involvement is detected in $15-20 \%$ of cases [8], while symptoms (blurred vision and decreased acuity) due to ocular 
involvement (vitreous/retina) are seen in 30\% and may precede or coexist with neurological symptoms [9].

Systemic B symptoms are unusual in patients with PCNSL.

Corticosteroids may induce a rapid improvement in clinical symptoms and radiographic features, and it is well known that at least $40 \%$ of the patients show steroid-induced responses. This point is important clinically but may be a disadvantage leading to false negative biopsies, thus delaying definitive diagnosis for months and even years. For this reason, until the diagnosis is established, corticosteroids should not be prescribed if there is a suspicion of PCNSL, unless severe worsening of patient's conditions occurs [4].

\section{PCNSL Diagnosis: Role of Standard and Advanced MRI}

Most common locations on MRI include periventricular white matter, basal ganglia, and corpus callosum [Figure 1], while cerebellum, brainstem, and spinal cord are less frequently involved [10]. In particular, spinal involvement is very uncommon and is seen in 3\% of patients with PCNSL. The most common intramedullary localization is the cervical cord, with solitary lesions in the majority of patients $[2,10]$. Due to the high cellularity, lesions are often hyperdense on CT and hypointense on T2-weighted MRI images with a variable amount of peritumoral edema [11, 12]. In most cases, the lesions enhance homogeneously [Figure 2(a)], but sometimes the enhancement is mild or with a ring pattern or is even absent [13]. Single lesions account for $70 \%$ of cases and multiple lesions for 30\%: a multifocal presentation is more frequent in immunocompromised patients.

The differential diagnosis is with high grade gliomas (HGGs) and less often TDLs (tumefactive demyelinating lesions), metastases (MTS), and infectious and granulomatous diseases [14].

3.1. PCNSL versus HGG. The most significant MRI difference between PCNSL and HGG is the pattern of enhancement: homogeneous in PCNSLs and heterogeneous with necrotic areas in HGGs. Furthermore, optic tracts and basal ganglia infiltration are more frequent in PCNSL, while cerebral cortex is affected more often in HGGs; nonetheless, "superficial" PCNSL variants mimicking HGG can be found $[15,16]$.

Many studies have explored diffusion-weighted imaging (DWI) findings in PCNSL and gliomas [15, 17-24]. PCNSLs have higher cellularity and nuclear-cytoplasm ratio than glioblastomas (GBMs). Thus, in PCNSL, diffusion is more restricted with lower apparent diffusion coefficient (ADC) in comparison to GBM. GBMs have a solid portion, which can show a restricted diffusion, but their cystic-necrotic areas display high water molecules diffusion [15, 17-19]. Some authors have shown that the relative minimum apparent diffusion coefficient (rADCmin) is the most accurate parameter to differentiate PCNSL from GBM $[22,25,26]$, and a cut-off value of 0.722 has been suggested, with $74.5 \%$ sensitivity and $74.1 \%$ specificity [26]. Another study [27] demonstrated that the initial area under the curve (IAUC)

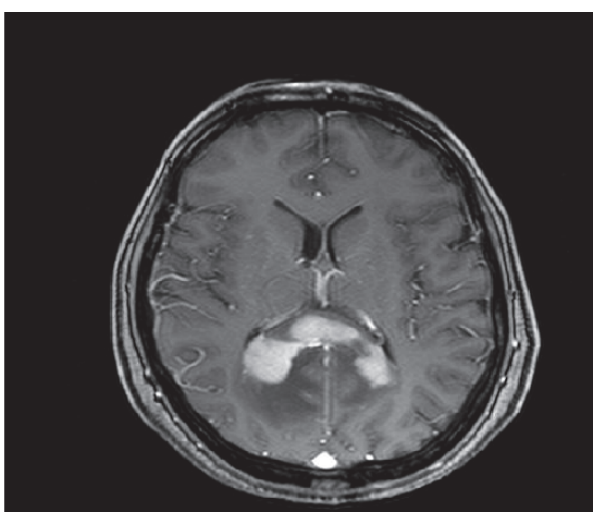

FIgURE 1: Contrast-enhanced axial T1-weighted MRI showing a PCNSL located in corpus callosum.

derived from dynamic contrast-enhanced MRI (DCE) may be a useful parameter together with ADC for differentiating PCNSL from atypical GBM (i.e., GBM with absent or limited necrosis), and thus the combination of quantitative ADC and permeability parameters from DCE MRI in tumor and peritumoral areas could help in discriminating PCNSL versus HGG [28].

${ }^{1} \mathrm{H}$-magnetic resonance spectroscopy $\left({ }^{1} \mathrm{H}\right.$-MRS $)$ can help in the differential diagnosis between PCNSL and GBM. Both tumors usually show reduced $\mathrm{N}$-acetylaspartate (NAA) peaks (neuronal damage), elevated choline to creatine ratio (elevated membrane turnover), lipid peaks (due to release of fatty moieties by transformed lymphocytes in PCNSL and due to necrosis in GBM), and lactate peaks (anaerobic metabolism). An increase in lipid resonance in the absence of necrosis seems to be the most specific finding in PCNSL [29-31] [Figures 2(b)-2(c)]. Nonetheless, given the difficulty in excluding small necrotic areas in many GBMs, other peaks have been investigated, such as glutamate (Glu) and glycine (Gln) [32]: Aburano et al. [33] reported that PCNSL shows higher Glu/Cr and Glu/Glu+Gln ratios than GBM.

Jiang et al. [34] investigated the usefulness of amide proton transfer weighted studies (APTW) in the diagnosis of PCNSL. APT is a technique that detects endogenous mobile proteins and peptides in tissue, such as those dissolved in cytoplasm. PCNSL has a high nuclear-cytoplasm ratio and prominent nucleoli and a relative low concentration of mobile proteins. APTW imaging in PCNSL shows homogeneous hyperintensities in enhancing areas, while HGGs have quite heterogeneous APTW hyperintensities, more often larger than the Gd-T1 lesions.

PCNSLs lack abundant neovascularization [35]: thus, on dynamic susceptibility contrast-enhanced MRI (DSCE$\mathrm{MRI}$ ), they show higher regional cerebral blood volume (rCBV) than normal tissue but lower rCBV when compared to the GBMs $[25,36,37$ ] [Figures 2(d)-2(e)]. Blasel et al. [38] demonstrated in PCNSL a typical "shoulder-like" pattern of signal intensity dynamics in most cases. Another study [39] identified a threshold in the rCBV value (2.56), which could be used to differentiate PCNSL from HGG with $>90 \%$ sensitivity and specificity and argued that the combination 


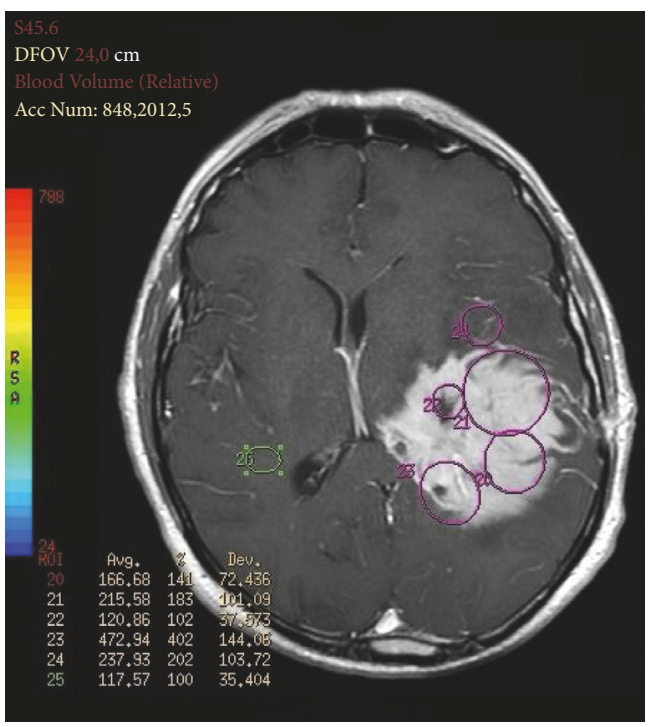

(a)

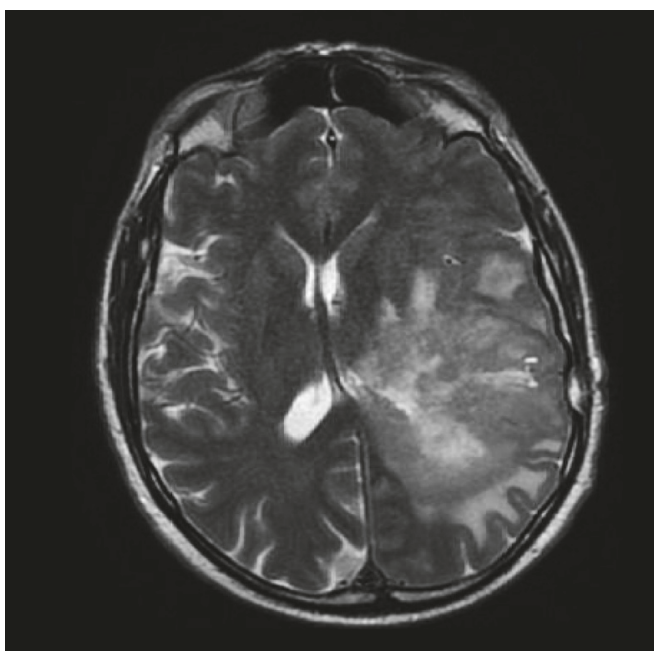

(b)

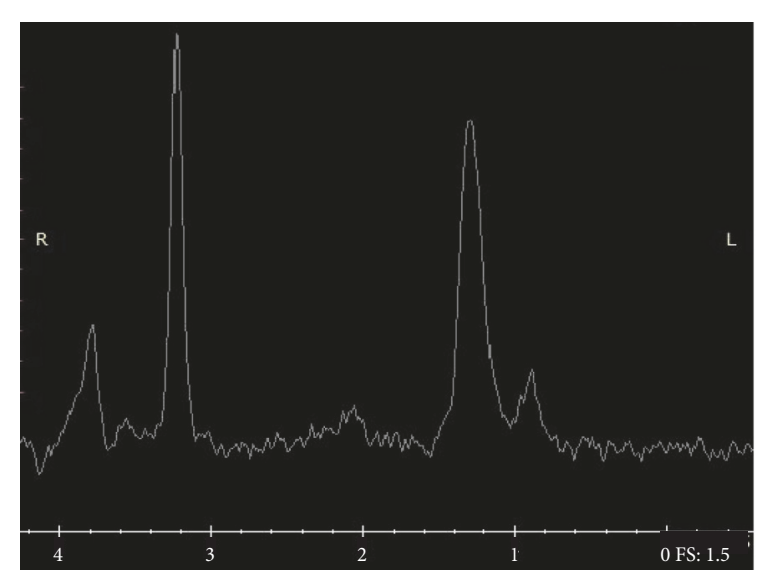

(c)

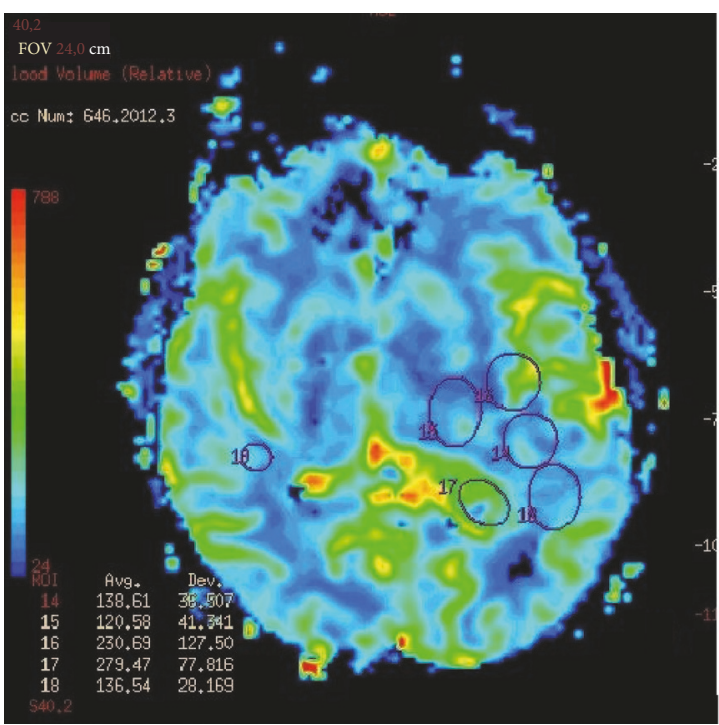

(d)

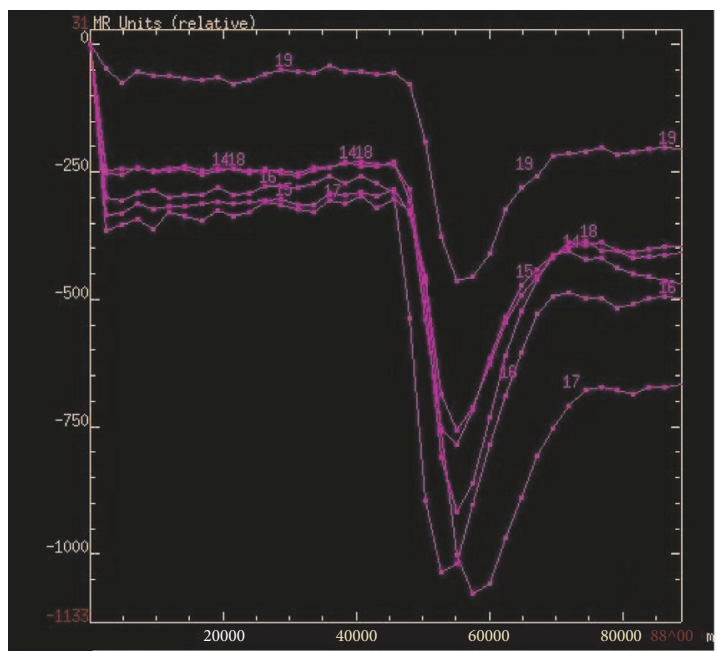

(e)

FIGURE 2: (a) Left temporoparietal PCNSL characterized by a homogeneous enhancing lesion on T1-weighted and (b) relatively low and inhomogeneous T2 signal on T2-weighted MRI. (c) Increased lipid peak on MRI spectroscopy and (d-e) increase of the regional cerebral blood volume (rCBV) when compared to the contralateral hemisphere. 
of $\mathrm{rCBV}$ and percentage of signal intensity recovery derived from DSCE-MRI could increase the accuracy of diagnosis.

3.1.1. Take Home Message. In the daily clinical practice, in addition to MRI morphological features, DSCE-MRI and MRS are the most reliable tools for a differential diagnosis and should be performed in particular in elderly patients when a surgical approach (biopsy versus resection) is to be decided.

3.2. PCNSL versus TDL. Tumefactive demyelinating lesions are sometimes another challenging differential diagnosis, especially in younger patients. On MRI, TDLs are solitary lesions, with T2-FLAIR hyperintense appearance, larger than $2 \mathrm{~cm}$ with mass effect and/or edema and variable enhancement patterns (homogeneous or heterogeneous, nodular or diffuse, punctate, open, or closed rings) [40]. Symptoms at initial presentation include headache, cognitive impairment, seizures, confusion, and impaired state of consciousness, which are common in PCNSL patients as well. Nonetheless, TDL patients have a clinical and radiological response to steroid therapy similar to that observed in PCNSL patients.

For a noninvasive diagnosis of TDLs, advanced imaging techniques are widely used. The typical DWI feature of TDLs is the heterogeneity of ADC values [41]. Myelin destruction and vasogenic edema can cause ADC increase [30, 42], but TDLs can also show reduced ADC values due to infiltration of inflammatory cells, especially in the periphery of the lesions [43]. Overall, TDLs consist of hypocellular lesions with ADC values higher than PCNSL, which conversely shows lower, homogeneous ADC values given the high cellularity [26, 44].

On DSCE-MRI, rCBV values are higher in PCNSL than TDLs [44]. MRS findings can be similar in PCNSL and TDL, consisting in decreased NAA (neuronal damage in PCNSL/axonal injury in TDL), increased choline (proliferation in PCNSL/myelin breakdown in TDL), lipid peak (fatty moieties released by transformed lymphocytes in PCNSL/membrane breakdown in TDL), and/or lactate peak (anaerobic metabolism in both entities) [45-47].

PCNSL may rarely be preceded by "sentinel demyelination," an entity characterized by histologically confirmed demyelinating inflammatory lesions, which mimics multiple sclerosis (MS) or acute disseminated encephalomyelitis (ADEM) [48-52]. It has been hypothesized that T-cell infiltrates, which can be found at biopsy, represent a cell-mediated immune response against the lymphoma, thereby masking the diagnosis of PCNSL $[53,54]$. Another hypothesis is that steroids, administrated prior to brain biopsy, disrupt B-cell lymphoma cells, whereas activated T-cells may be relatively protected from steroid-induced apoptosis [54]. In this regard, a recent retrospective study examining approximately 1000 cases of PCNSL has reported that the effects of corticosteroids given before the biopsy rendered an accurate diagnosis difficult in up to $50 \%$ of cases [55].

Red flags suggesting PCNSL include advanced age, worsening of clinical conditions despite treatment for a demyelinating disease, and lesions progression on neuroimaging studies over time. Conversely, MS onset in old patients is often characterized by spinal cord involvement [49]. To improve the differential diagnosis between demyelinating diseases and PCNSL, tools such as visual evoked potentials, spinal cord MRI, and cerebrospinal fluid (CSF) examination for oligoclonal bands should always be performed. Moreover, especially in younger patients, a search in the past history for minor episodes of subacute visual or neurological deficits with spontaneous resolution, suggestive of a first MS relapse, should be performed.

3.2.1. Take Home Message. In the daily clinical practice, this diagnostic problem is rare; however, when present, a combination of radiological, neurophysiological, and laboratory findings and the evaluation of their changes over time are needed.

3.3. PCNSL versus MTS. The typical localization of metastases includes the cortical gray-white matter junction of cerebral hemispheres, cerebellum, and basal ganglia in decreasing order. On MRI brain MTS can be solitary (40\%) or multiple $(60 \%)$, often with central necrosis, a peripheral ring of enhancement, and extensive edema [56-58]. On DWI, metastases show reduced diffusivity in the peripheral enhancing part and increased diffusivity in the central necrotic area and surrounding edema, while PCNSLs show a homogeneous reduced diffusivity due to the high cellularity [59]. rCBV is lower in PCNSL than metastases, due to lack of neovascularization. MRS studies investigating differences between PCNSL and metastases are not available.

3.3.1. Take Home Message. In the daily clinical practice, when multiple lesions are evident on MRI, a differential diagnosis between brain metastases and PCNSL should always be taken in mind.

3.4. PCNSL versus Infectious and Granulomatous Diseases. In AIDS patients, a differential diagnosis between PCNSL and Toxoplasmosis Encephalitis (TE) is not always straightforward, since these two entities can show similar clinical findings and mass lesions on imaging. Clinically, patients with TE can present either acutely or insidiously with variable combination of headache, fever, encephalopathy, seizures, and focal deficits. On MRI, TE lesions are generally isointense to hypointense on T1-weighted images with ring or nodular enhancement, while in T2 they appear as hyperintense (during liquefactive necrosis), hypointense (in the posttreatment phase), or isointense, depending on the stage and composition of the abscess. They are often located in basal ganglia or in the junction between the white and gray matter of cerebral hemispheres and less commonly in the brainstem. Extensive T2-hyperintense vasogenic edema is typically present. Toxoplasma encephalitis lesions are multifocal on MRI in $86 \%$ of cases [60].

In DWI studies, TE lesions show higher $\mathrm{ADC}$ values than PCNSL [61]. The ability of MRS to differentiate TE from PCNSL depends on the voxel placement over necrotic or cellular areas and on the lesion stage. Nonetheless, in 
typical cases, lipid/lactate peaks (reflecting anaerobic, necrotizing inflammatory process) are present without elevated Cho/Cr ratio typical of PCNSL. Last, rCBV values are substantially higher in PCNSL as compared to TE lesions [62].

Neurosarcoidosis can present with multiple enhancing brain lesions mimicking PCNSL. Magnetic resonance imaging findings are variable. About $40 \%$ of patients with neurosarcoidosis have either leptomeningeal enhancement or multiple white matter enhancing lesions [63]. In these cases, differential diagnosis should be based on serum ACE levels, CSF examination (usually with pleocytosis, increased protein level, and oligoclonal bands), chest high-resolution CT (HRCT), and bronchoalveolar lavage (BAL). Nonetheless, biopsy of suspected granulomatous lesions is the gold standard for diagnosis [64].

3.4.1. Take Home Message. In the daily clinical practice, this diagnostic problem is rare. However, patient history, CSF, and serum analysis are fundamental to exclude the hypothesis of infectious diseases. In particular CSF examination, ACE levels and HRTC are needed to exclude the hypothesis of neurosarcoidosis.

\section{PCNSL Diagnosis: Role of PET}

In 1992, Rosenfeld et al. reported a strong ${ }^{18} \mathrm{~F}$-Fluorodeoxyglucose (18F-FDG) uptake in a group of 10 patients with PCNSL [65]. These characteristics depend on the high cellular density and increase glucose metabolism: the semiquantitative ${ }^{18}$ F-FDG uptake values, measured by maximum standardize uptake value (SUVmax), are reported to be 14-22 in PCNSL, and this value is about 2.5 times higher than the average SUV in the normal gray matter [66]. However, the high uptake of basal ganglia, cerebral cortex, and thalamus makes the diagnosis problematic.

${ }^{18}$-FDG PET can play a role for diagnosis in patients who cannot undergo brain biopsy due to surgical risks, older age, or comorbidities. Yamaguchi et al. [67] demonstrated that inoperable PCNSL can indirectly be diagnosed with good accuracy on the basis of some PET-MRI criteria: extremely high tumor FDG uptake relative to normal gray matter and tumor reduction 1 week after corticosteroid administration on contrast-enhanced T1-weighted MRI.

Total body ${ }^{18}$ F-FDG-PET has an important role in PCNSL staging at diagnosis or in the follow-up, as it can diagnose a systemic disease with higher sensitivity than conventional imaging. Mohile et al. [68] showed that $7 \%$ of patients with suspected PCNSL were found to have systemic NHL by total body FDG-PET, while body CT scans and bone marrow biopsies were negative. Moreover, total body FDGPET was positive in $27 \%$ of patients during restaging for recurrent disease.

Regarding amino acid PET, methionine (MET) uptake reflects an increase of amino acid transport and protein synthesis and is related to cellular proliferation. In the evaluation of brain lesions, MET has some advantages over
FDG, stemming primarily from a low uptake in the normal brain. MET is thought to be useful for delineating tumor boundaries of PCNSL, with the area of increased uptake being larger than the enhancing lesions on MRI [69]. Kawase et al. [70] did not find significant differences between $\mathrm{T} / \mathrm{N}$ (tumor to normal contralateral cortex activity) ratios on MET-PET and FDG-PET, although mean values of SUV on MET-PET in CNS lymphomas were significantly lower than those on FDG-PET. In this case series, 2 of 13 FDG-PET scans did not show a marked accumulation in the tumor (small disseminated lesions in one case and disseminated lesions overlaying the cortex in the other case), while increased MET uptake was observed in both patients.

Nonetheless, the experience in the use of amino acid PET in patients with PCNSL is still limited.

Take Home Message. The typical PCNSL metabolic pattern is an area of high homogeneous increase of ${ }^{18} \mathrm{~F}-\mathrm{FDG}$, more often in subcortical regions. Frequently, this area is more visible at visual analysis due to the presence of hypometabolism in the adjacent cortex because of compression or edema phenomena.

These patterns are maintained in the disease recurrence as well [Figure 3].

4.1. PCNSL versus GBM or MTS. The ${ }^{18} \mathrm{~F}-\mathrm{FDG}$ uptake in PCNSL is usually homogenous in contrast to the inhomogeneous uptake in GBMs and metastases. Kosaka et al. [71] identified maximum standard uptake values (SUVmax) on FDG-PET as the most important parameter for distinguishing lymphomas from other brain tumors. They used a SUVmax of 15 as a cut-off for diagnosing CNS lymphoma, and only one high grade glioma yielded a false-positive result. Based on these findings, Makino et al. [72] demonstrated that the accuracy of FDG-PET for differentiation of PCNSL versus GBMs and metastases was 0.86 when the SUVmax cut-off value was set at 12 with sensitivity of $100 \%$ and specificity of $71.4 \%$.

Yamaguchi et al. [67] showed that FDG uptake using the $\mathrm{T} / \mathrm{N}$ ratio was more reliable than SUVmax, since SUVmax is influenced by plasma glucose levels. The appropriate $\mathrm{T} / \mathrm{N}$ ratio cut-off point was 2.0 for differentiating PCNSL (T/N ratio $>2$ ) from other malignancies (GBMs and metastases) when patients were not on corticosteroids. FDG uptake could be influenced by cumulative doses of corticosteroid before a PET scan, a point that should always be considered. A recent meta-analysis [73] on ${ }^{18} \mathrm{~F}-\mathrm{FDG}$ PET and PET/CT in PCNSL, based on 8 retrospectives studies (129 patients), revealed pooled sensitivity and specificity of ${ }^{18}$ F-FDG-PET and PET/CT in the diagnosis of PCNSL of 0.88 and 0.86 , respectively.

Okada et al. [69] suggested higher FDG-PET SUVmax and higher $\triangle$ SUVmax (ratio of SUVmax in the late and early phase) on MET-PET for PCNSL compared with GBM.

Take Home Message. SUVmax is influenced by different factors, in particular the sensitivity of the PET scanner. 


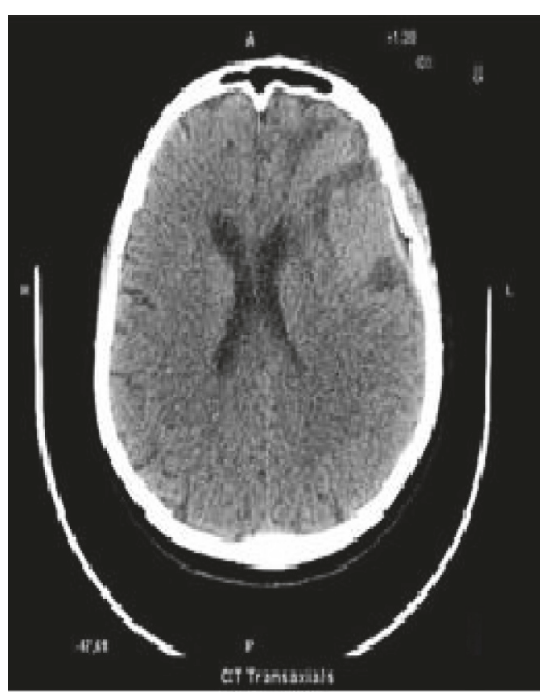

(a)

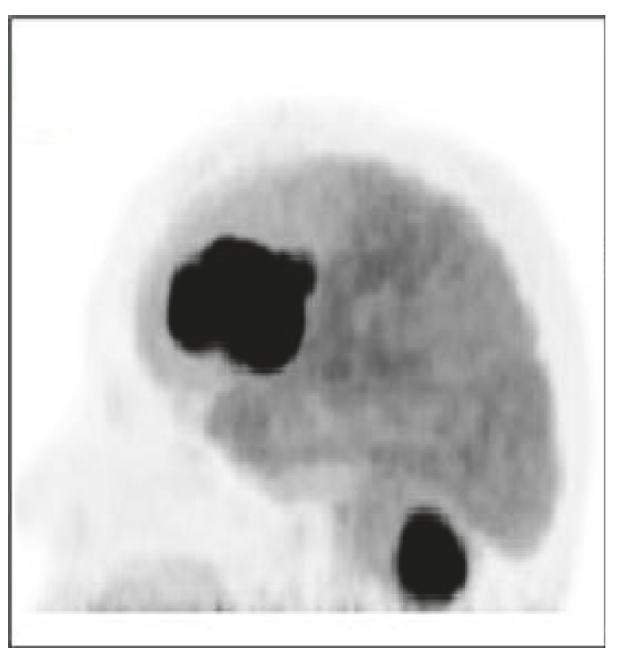

(d)

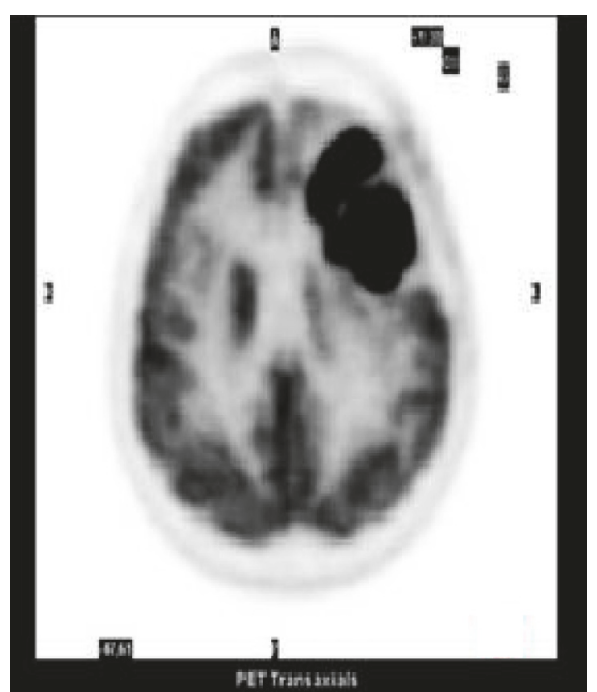

(b)

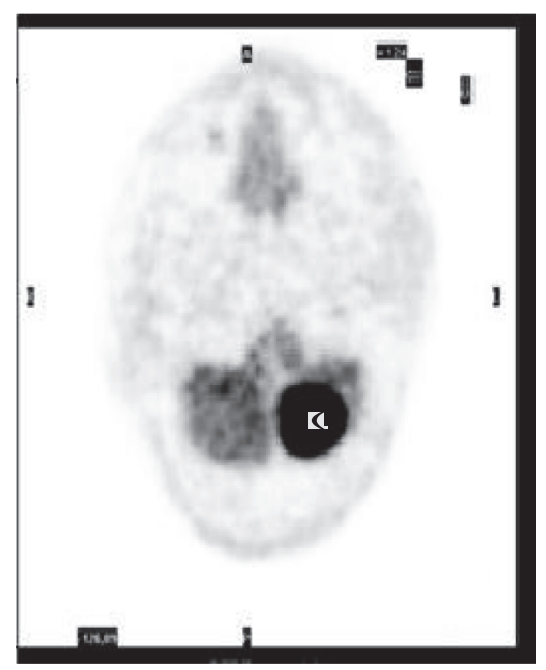

(e)

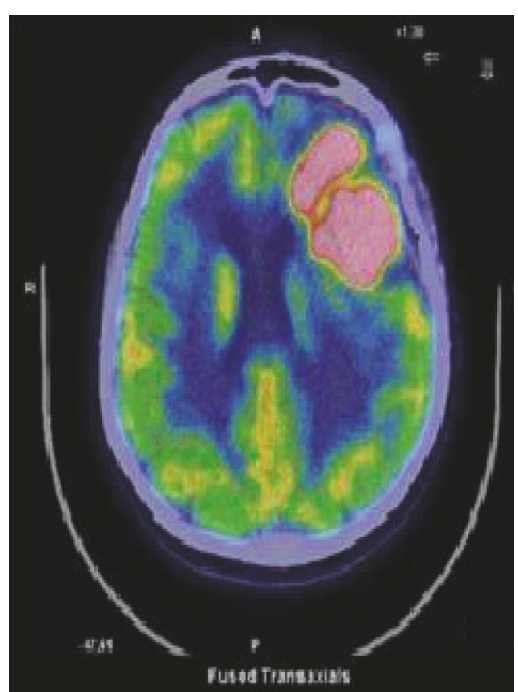

(c)

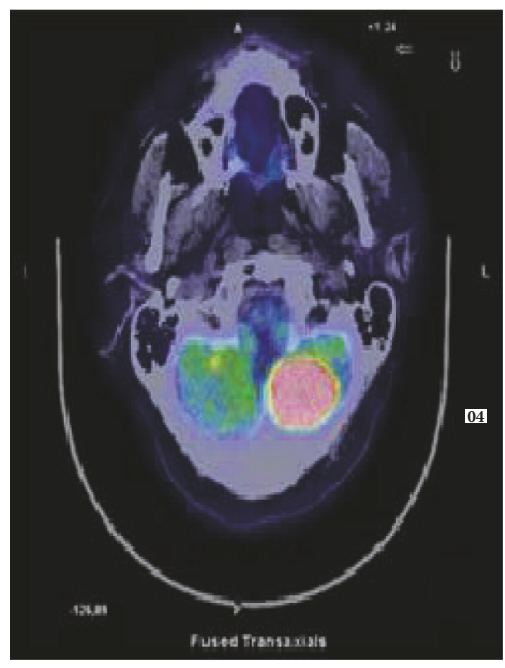

(f)

FIGURE $3:{ }^{18}$ F-FDG brain imaging in a 66-year-old woman with PCNSL. Axial CT (a), PET (b), and PET/CT fusion image (c) showing the FDG-avid lesion involving the left frontal lobe (SUVmax 42). (d) Maximum imaging projection (MIP), axial CT (e), and PET/CT fusion image (f) showing another lesion on the left cerebellar hemisphere.

Therefore, particular attention must be paid when the values of this parameter are imported from literature into own clinical routine.

4.2. PCNSL versus TDL. Schiepers et al. [74] reported that active lesions in acute multiple sclerosis are hypermetabolic, while chronic lesions are hypometabolic: thus, glucose uptake could be a marker of the temporal stage of a plaque. Takenaka et al. [75] studied 6 cases of TDLs and found a mean FDG T/N ratio similar to the glucose metabolism of normal cortex and lower than those of GBMs. The same authors described a lower MET uptake in TDL than in glial malignancies. Padma et al. [76] reported low glucose uptake and prominent methionine uptake in a case of TDL: the authors hypothesized that MET uptake in the lesion was related to inflammation and blood-brain barrier disruption. Maffione et al. [77] described moderate focal glucose uptake in two TDLs (SUVmax 6.9 in both cases). Overall, studies comparing ${ }^{18} \mathrm{~F}$-FDG PET and MET-PET findings in PCNSL and TDLs are lacking. Patients with demyelinating diseases can show diffuse cortical hypometabolism due to chronic white matter damage $[78,79]$, especially in patients with subacute/chronic neurological impairment and small white matter lesions. However, cortical hypometabolism does not exclude a diagnosis of lymphoma, as it can be an expression of a cortical disconnection due to white matter lesions (tumoral or not). The use of MET-PET could more precisely indicate the presence of a tumoral lesion [80].

Take Home Message. In case of negative or doubtful results at 1 hour ${ }^{18} \mathrm{~F}$-FDG postinjection images, it is advisable to repeat a 
delayed PET acquisition at 4 hours after injection. In some cases, this procedure allows an improvement in the visual analysis.

4.3. PCNSL versus Infectious and Granulomatous Diseases. In patients with acquired immunodeficiency syndrome (AIDS),

${ }^{18}$ F-FDG uptake can be used to distinguish between cerebral PCNSL (highly metabolic lesions) and toxoplasmosis (hypometabolic lesions) [81].

Neurosarcoidosis can be hypermetabolic on brain ${ }^{18} \mathrm{~F}$ FDG-PET [82]. In suspected neurosarcoidosis, whole-body FDG-PET can help in localizing granulomatous lesions, which can be a target for biopsy [82]. MET-PET yields similar results. Kawai et al. [66] described a series of nontumoral lesions with moderate MET uptake, including brain abscesses. $\mathrm{Ng}$ et al. [51] demonstrated MET uptake and high FDG uptake in multiple brain lesions due to neurosarcoidosis. These results are not surprising, since MET uptake can be increased as a result of increased density of inflammatory cells and disruption of the blood-brain barrier (BBB).

Take Home Message. ${ }^{18}$-FDG-PET should be performed, together with MRI spectroscopy and perfusion MRI, when suspecting a PCNSL prior to steroid treatment and biopsy. To date, the major limitation of ${ }^{18}$ F-FDG-PET is the difficulty to diagnose PCNSLs with atypical radiological findings (disseminated or nonenhancing lesions) [Figure 4]. Further studies are needed to determine the diagnostic value of MET uptake and the usefulness of combining MET with FDGPET. Nonetheless, the ability of MET-PET to detect PCNSL in disseminated or cortical lesions suggest a role in the diagnosis of suspected PCNSL with atypical findings.

\section{PCNSL Diagnosis: Role of CSF}

A recent review on the accuracy of flow cytometry (FCM) and cytomorphology (CM) in the diagnosis of meningeal involvement from lymphoid neoplasms [83], based on 27 studies, demonstrated a great heterogeneity: positive results with both FCM and CM range from $0.3 \%$ to $42.9 \%$ among studies. Samples with positive FCM but negative CM are reported by $89 \%$ of the studies, while samples with positive CM and negative FCM are found in $48 \%$. In a study by Schroers et al. [84] regarding a cohort of PCNSL patients, $23.3 \%$ were positive for CSF lymphoma cells with flow cytometry in contrast to $13.3 \%$ patients with positive cytopathology. It must be noted that CSF cells are particularly fragile and must be analyzed in the hours following the examination or be put in a stabilizing suspension. Overall, one can suggest employing flow cytometry along with conventional cytology to increase the number of positive CSF cells. Another method to detect mature B lymphoid cells is PCR testing for IgH gene rearrangements, which does not require intact cells [85].

Studies regarding the diagnostic role of different CSF markers (interleukins, chemokines, and receptors) and miRNAs are ongoing. Interleukin-10 (IL-10) and its receptors are expressed in PCNSL and act as a growth factor for B lymphocytes, while IL-6 is related to lymphoid cells growth and immunity regulation. Sasayama et al. [86] demonstrated that CSF IL-10 and IL-6 levels are significantly higher in PCNSLs than in other brain tumors: at an IL-10 cut-off level of $9.5 \mathrm{pg} / \mathrm{mL}$, the sensitivity and specificity were $71.0 \%$ and $100 \%$, respectively. Nguyen-Them et al. [87] reported that IL-10 CSF concentration can distinguish PCNSL from other neurologic diseases with sensitivity of $88.6 \%$ and specificity of $88.9 \%$ with a cut-off of $4 \mathrm{pg} / \mathrm{ml}$, while Song et al. [88] reported diagnostic sensitivity and specificity of $95.5 \%$ and $96.1 \%$, respectively, when IL-10 levels cut-off was set at $8.2 \mathrm{pg} / \mathrm{ml}$.

Osteopontin $(\mathrm{OPN})$ is another proinflammatory cytokine involved in immune cell activation and B-cell migration and proliferation. Strehlow et al. [89] demonstrated that CSF OPN levels in PCNSL patients are significantly higher (> $620 \mathrm{ng} / \mathrm{mL}$ ) than those in patients with inflammatory CNS disease and GBM or in healthy controls. Similarly, Viaccoz et al. [90] reported that CSF neopterin (a marker of neuroinflammation) levels are significantly higher in patients with PCNSL than in those with other brain tumors, pseudotumoral inflammatory brain lesions, or nontumefactive inflammatory CNS disorders, with 96\% sensitivity and 93\% specificity for the diagnosis of PCNSL.

5.1. Take Home Message. Combined "classical" CSF analysis (i.e., CM and CFM) increases the diagnostic accuracy for PCNSL, but a role for CSF markers of B lymphoid cell proliferation is emerging. These new biomarkers could improve the differential diagnosis of PCNSL but need to be validated and standardized before a routine application in clinical practice takes place.

\section{PCNSL Diagnosis: Role of Ocular Examinations}

When PCNSL is suspected, ocular evaluation including fundoscopy and slit lamp examination should be performed. Ocular involvement must be confirmed by vitreous biopsy, and a positive result in a context of brain MRI suspicious for PCNSL can be diagnostic and avoid brain biopsy. A positive cytology is obtained in $50 \%$ of cases; as for CSF, immunophenotyping and detection of $\mathrm{IgH}$ or T-cell receptor rearrangements by PCR analysis indicating monoclonality are helpful tools for diagnosis. Moreover, high levels of IL10 and/or high IL-10/IL6 ratio in ocular fluids are strongly suggestive of B-cell lymphomatous uveitis [9]. Fluorescein angiography may be useful for lymphomatous involvement of the retina.

6.1. Take Home Message. A cytological confirmation of ocular involvement should be performed whenever feasible.

\section{PCNSL Diagnosis: Role of Baseline Cognitive Assessment}

Cognitive symptoms are critical domains to assess in PCNSL patients, since they can prevail at diagnosis. Neuropsychological baseline testing is fundamental to allow a better 


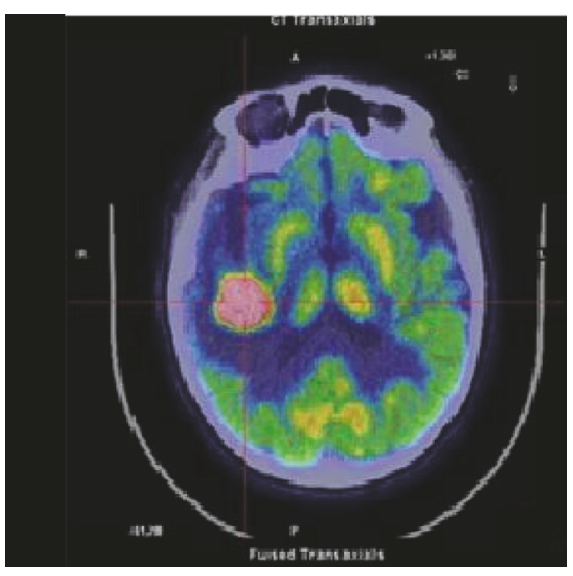

(a)

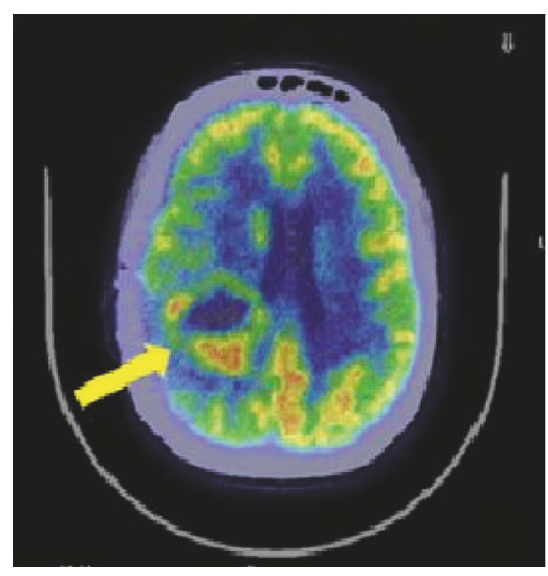

(b)

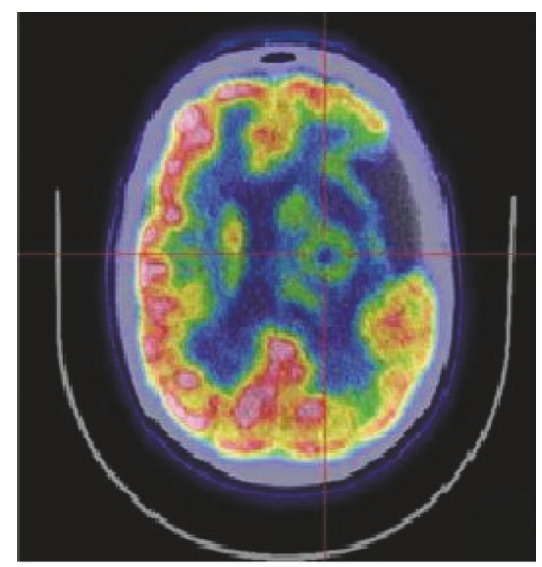

(c)

Figure 4: Axial ${ }^{18}$ F-FDG brain imaging. PCNSL (a), glioblastoma multiforme (b), and abscess (c).

interpretation of cognitive decline following therapy and in follow-up phases. Guidelines from Correa et al. [91] outlined the importance of standardization of neuropsychological evaluation in PCNSL: a battery of tests to assess attention, executive function, verbal memory, motor function (domains frequently impaired in PCNSL patients), quality of life, and premorbid IQ was proposed.

Cognitive assessment may sometimes help in distinguishing PCNSL from other nonneoplastic diseases (e.g., Creutzfeldt-Jakob disease, autoimmune encephalitis, paraneoplastic encephalitis, and infectious conditions): in PCNSL patients, memory impairment is more marked than in other rapidly progressive dementias (RPDs), while neurological signs, such as myoclonus and parkinsonism, are very rare [92].

7.1. Take Home Message. Cognitive tests should be performed in PCNSL patients before any antineoplastic therapy.

\section{PCNSL Prognostication: Role of MRI and PET}

Recent studies suggest that some parameters of advanced MRI can predict patients' prognosis and tumor responsiveness to methotrexate-based chemotherapy. Zhang et al. [93] investigated ADC as a prognostic indicator in 28 patients with PCNSL receiving high-dose methotrexate-based chemotherapy: patients with higher ADC 5\% (ADC at 5\% percentile) showed significantly longer progression-free survival (PFS). Similarly, Barajas et al. [94] showed an inverse correlation between cellular density and ADC measurement: in particular, $\mathrm{ADC} 25 \%$ values (ADC at $25 \%$ percentile) less than the median value of 692 ("low ADC group") were associated with significantly shorter progression-free survival (PFS) and overall survival (OS).

Wieduwilt et al. [95] reported similar results, demonstrating shorter PFS and OS in patients with lower ADCmin values (less than $384{\mathrm{X} 10^{-6}}^{\text {) }}$ prior to immunochemotherapy.
Valles et al. [96] reported that PCNSL patients with low ADCmin or low rCBV values have worse PFS and OS and hypothesized that low tumor rCBV in PCNSL could express a lack of tumor angiogenesis and a decrease in the number of vessels able to deliver intravenous methotrexate to the tumor. In line with these findings, Chung et al. [97] showed that the pattern of DCE-MRI could be a marker for predicting complete response (CR) and longer PFS following chemotherapy, with a strong association between "diffuse" DCE-MRI pattern and CR. Regarding ${ }^{18}$ F-FDG-PET, Kawai et al. [98] demonstrated that pretreatment ${ }^{18}$ F-FDG uptake can predict survival in PCNSL patients: the median survival time of patients with low-to-moderate ${ }^{18} \mathrm{~F}$-FDG uptake (SUVmax < 12) was significantly longer (26 months) than that of patients with high ${ }^{18}$ F-FDG uptake (SUVmax $\geq 12 ; 12$ months).

8.1. Take Home Message. Prognostication by MRI and PET is thus far limited to clinical trials. Nonetheless, a correlation between lower ADC (reflecting high tumor cellularity), lower rCBV (reflecting poor vascularity), higher FDG metabolism, and relatively poorer response to treatment can be hypothesized.

\section{PCNSL Prognostication: Role of CSF}

CSF IL-10 levels decrease after PCNSL treatment [86, 87] and increase at time of relapse [86]. High baseline and posttreatment IL-10 levels have been associated with poor PFS [86-88]. Moreover, CSF OPN levels have been associated with shorter PFS and OS [89]. Other potential biomarkers of PCNSL, related to disease response to therapy, include CSF levels of transmembrane activator and CAML interactor (TACI), soluble CD19 [99], antithrombin III [100], free immunoglobulin light chains [101], CXCL13 [102], and miRNAs. With regard to the latter, Baraniskin et al. [103] demonstrated the ability of CSF miR-21, miR-19b, and miR92 levels to differentiate PCNSL from other nonneoplastic 
neurological disorders and correlate with different phases of PCNSL disease. No role was demonstrated for serum miRNA levels.

9.1. Take Home Messages. Prognostication by CSF markers is thus far limited to clinical trials.

\section{PCNSL Treatment: Monitoring of the Disease}

Standard treatment of PCNSL includes 2 phases: induction and consolidation. Induction strategies usually consist in high-dose methotrexate- (HD-MTX-) based polychemotherapy: combination of HD-MTX with other CT agents could improve response compared with HD-MTX alone. Drugs found to be useful and safe when combined with HDMTX are cytarabine, lomustine, procarbazine, vinca alkaloids, temozolomide, and thiotepa [104]. HD-MTX-based polychemotherapy can be associated with the administration of rituximab (RTX), an anti-CD-20 monoclonal antibody $[4,105]$.

Induction phase is followed by consolidation treatments, such as whole-brain radiotherapy (WBRT), high-dose myeloablative chemotherapy supported by autologous stemcell transplantation (HDC-ASCT), and nonmyeloablative chemotherapy. WBRT was for long time used with doses between 40 and 45 Gy in 20-25 fractions, but these doses were associated with important cognitive dysfunctions: for this reason, reduced-dose (RD) WBRT $(23.4 \mathrm{~Gy})$ is commonly used. A recent phase III study by Thiel et al. [106] suggested that consolidation with RD-WBRT can improve patients' PFS but not OS. On these bases and considering the risk of neurotoxicity related to RT, consolidation strategies to minimize neurotoxicity have been proposed, such as avoiding WBRT in patients with CR after chemotherapy, lower doses of WBRT, and replacing consolidation radiotherapy with other treatments such as ASCT $[107,108]$.

A recent International Extranodal Lymphoma Study Group-32 (IELSG32) trial proposed the regimen MATRix (a combination of HD-MTX, HD-ARAC [cytarabine] thiotepa, and rituximab) as new standard chemoimmunotherapy [109]. Treatment regimens based on CT (i.e., HD-MTX alone or associated with other chemotherapeutic agents such as temozolomide) have been suggested as a reasonable approach for elderly patients with good PS and renal function [110-112].

Patients with recurrent/refractory disease typically have poor outcomes, with response rates ranging between 30 and $60 \%$ and PFS ranging between 2 and 6 months. The key points in relapsed disease are MTX sensitivity and performance status of the patient. In cases with MTX-sensitive relapse, additional MTX cycles must be administered to achieve maximal cytoreduction (6-8 cycles). This must be followed by dose-intensive CT consolidation in "fit patients," CNS penetrant agents such as thiotepa, or carmustinebased regimens. Topotecan, temozolomide, pemetrexed, bendamustine, PCV, ifosfamide-etoposide-based regimen, and cisplatin-cytarabine-based regimens are feasible choices for salvage treatment. However, a standard of care thus does not exist so far. The most appropriate salvage treatment should be chosen on the basis of patient's age, PS, comorbidities, site of relapse, previous therapy, and duration of previous response and expected side effects of salvage drugs. HDCT with ASCT is an option in selected relapsed/refractory cases, but there is evidence of superiority of this strategy to chemoradiotherapy [4].

New targeted agents, such as lenalidomide and ibrutinib, are showing preliminary interesting results in relapsed CNS lymphoma $[113,114]$. Other promising therapeutic agents are the "check-point inhibitors" of PD-1/PD-L1 pathway, which play a crucial role in tumor immunology [115]. PD-1/PDL1 expression has been recently detected in $42 \%$ of the lymphomas in a large cohort of 10.187 PCNSL samples [116].

\section{MRI and PET: What to Value and When}

The International Primary CNS Lymphoma Collaborative Group defined in 2005 the guidelines for evaluating PCNSL response to treatments based on the evaluation of gadolinium enhancement on MRI, use of corticosteroids, eye examination, and CSF cytology. Combining these elements, patients' response to therapy can be defined as complete response (CR), unconfirmed complete response (uCR), partial response (PR), stable disease (SD), or progressive disease (PD) [117].

Patients enrolled into clinical trials should be assessed after the completion of therapy by brain imaging at a minimum of 3 months for 2 years and then every 6 months for 3 years and yearly for at least 5 years. A recent study by Fossard et al. [118] aimed to define the best follow-up strategy for PCNSL patients after first-line therapy in clinical practice. 125 PCNSL patients, who achieved CR after firstline therapy, started the follow-up and the timing of planned visits and MRI was defined by each center. Overall, serial imaging detected relapses in a minority of asymptomatic patients only. No differences in patient outcome between symptomatic and asymptomatic relapses were seen. Tabouret et al. [119] investigated the prognostic value of conventional MRI characteristic and defined tumor responsiveness to chemotherapy. In a cohort of 85 PCNSLs, infratentorial location was associated with shorter OS and larger enhancing tumor volume correlated with poor PFS; moreover, the percentage of decrease in $\mathrm{T} 1$ enhancement between baseline and first MRI evaluation correlated with OS. These authors also described at baseline T2-FLAIR hyperintense lesions distant from the enhancing tumor in $26 \%$ of patients: $89 \%$ of these lesions decreased after chemotherapy, and in $50 \%$ of patients relapses originated from these areas, reinforcing the hypothesis of their neoplastic nature. These data underline the difficulty of interpretation, especially after treatment, of T2-FLAIR hyperintense nonenhancing alterations, which can be variably attributed to vascular damage, leukoencephalopathy related to MTX or radiotherapy, or neoplastic tissue.

Advanced MRI techniques and FDG-PET or MET-PET could improve the prediction of response, progression, and outcome. A reduction of ADC values after chemotherapy 


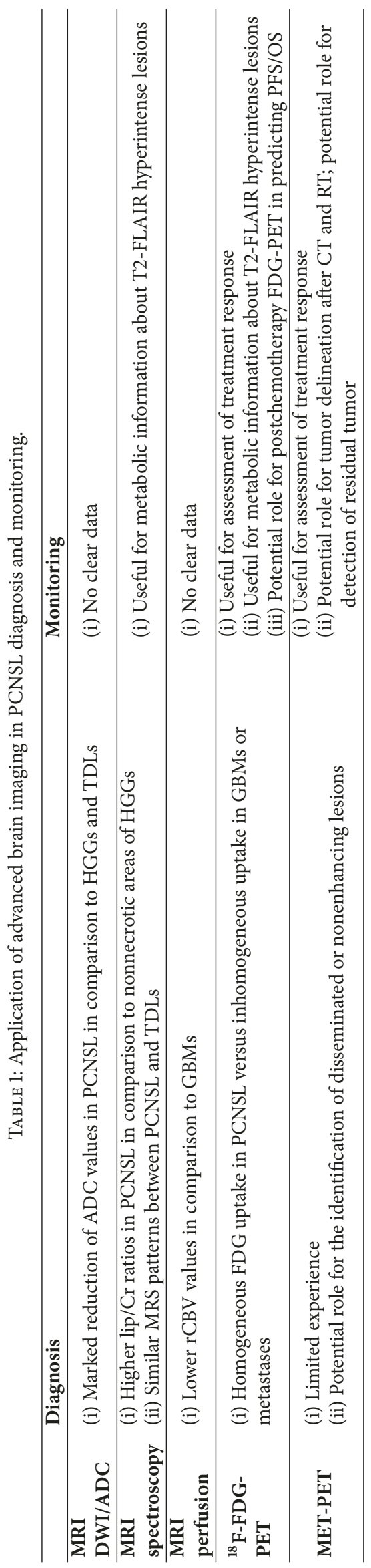


correlated with methotrexate responsiveness [93]. Palmedo et al. [120] showed that FDG-PET was able to predict complete remission or tumor recurrence after chemotherapy in 8 patients affected by PCNSL. Jo et al. [121] showed that patients with negative FDG-PET after chemotherapy had significantly longer progression-free survival, but not overall survival, compared to the group with positive posttreatment PET. Conversely, Mercadal et al. [122] detected a difference in overall survival between negative and positive posttreatment FDG-PET patients (100\% versus $37.5 \%$, resp., $p=0.045)$. Some authors have hypothized that the reduction of ${ }^{18} \mathrm{~F}-$ FDG uptake after the first cycles of chemotherapy could precede the reduction of tumor size on MRI [98]. Thus, the combination of ${ }^{18}$ F-FDG-PET and MRI could improve the evaluation of treatment response [123] and the appreciation of residual MRI lesions during follow-up examinations. [122]. MET-PET could be useful for an early delineation of response to systemic chemotherapy or radiotherapy [124].

In conclusion, FDG-PET can be used to evaluate treatment response of PCNSL at a very early stage and predict a more aggressive disease course. During long-term follow-up, FDG-PET findings could anticipate the diagnosis of PCNSL recurrence, even if this hypothesis should be demonstrated in prospective studies with higher number of patients. METPET may have a role in identifying residual tumor that is difficult to detect by MRI owing to the effects of previous radiotherapy or surgery [Table 1].

11.1. Take Home Message. In the daily clinical practice, monitoring of response/progression with enhanced MRI is mandatory, while advanced MRI techniques and PET imaging are confined to clinical trials.

\section{Cognitive Monitoring}

Serial monitoring of cognitive functions is important for two reasons. First, cognitive impairment can be a marker of recurrence. Fossard et al. [118] reported that $80 \%$ of PCNSL relapses were not detected by MRI examinations but on the basis of neurological symptoms, in particular cognitive impairment in $43 \%$ of patients. Second, cognitive followup is crucial to assess the long-term effects of treatments, in particular central neurotoxicity. Clinically, neurotoxicity presents as a progressive cognitive impairment, which can lead to dementia, and develops after a variable delay from the end of treatment. Cognitive dysfunctions can parallel or follow motor and autonomic symptoms. Clinical features mimic diseases, such as Binswanger dementia or normal pressure hydrocephalus [125]. Risk factors for neurotoxicity include older age, comorbidities, leptomeningeal disease, WBRT, and aggressive chemotherapy regimens. Several chemotherapeutic agents, particularly HD-MTX and highdose cytarabine, have been shown to cause periventricular white matter abnormalities [126]. Combined regimens, including WBRT, display a higher percentage of neurotoxicity when compared to chemotherapy alone, with an incidence of neurotoxicity ranging from $8 \%$ to $50 \%$ of patients [126-132]. Combined modality treatments, including RT, are associated with cognitive impairment even in patients below 60 years of age [133], but the frequency is higher above 60 years of age. For this reason, many authors suggest to defer WBRT at recurrence in older patients [134-136]. Encouraging results are coming from trials on HDC-ASCT, with no significant neurotoxicity and even improved cognitive functions and/or quality of life $[108,125]$.

The possibility to derive information on treatments neurotoxicity depends on cognitive testing of patients in remission, since in cases of PCNSL progression the cognitive decline depends on the disease itself [91].

12.1. Take Home Message. In daily clinical practice, cognitive testing should be encouraged to better define the cognitive profile in the different scenarios (persistent response versus disease recurrence).

\section{Conclusions}

The knowledge of neuroimaging and biological markers of PCNSL is rapidly growing. Thus, it is conceivable that in future years the scenario of PCNSL management will change, moving to a better disease-profiling and "tailoredon-patient" therapies. Cooperation and scientific dialogue between different medical professionals (neurologists, neuroradiologists, nuclear medicine physicians, hematologists, oncologists, neurosurgeons, ophthalmologists, pathologists, and psychologists) are essential to improve diagnosis, prognosis, treatment strategies, and outcomes of such a complex disease in both clinical trials and everyday clinical activity.

\section{Conflicts of Interest}

The authors do not have any conflicts of interest to declare.

\section{References}

[1] J. L. Villano, M. Koshy, H. Shaikh, T. A. Dolecek, and B. J. McCarthy, "Age, gender, and racial differences in incidence and survival in primary CNS lymphoma," British Journal of Cancer, vol. 105, no. 9, pp. 1414-1418, 2011.

[2] J. Carnevale and J. L. Rubenstein, "The challenge of primary central nervous system lymphoma," Hematology/Oncology Clinics of North America, vol. 30, no. 6, pp. 1293-1316, 2016.

[3] E. Montserrat, "Primary CNS lymphoma: in search of the evidence," The Lancet Haematology, vol. 2, no. 6, pp. e227-e228, 2015.

[4] S. Paydas, "Primary central nervous system lymphoma: essential points in diagnosis and management," Medical Oncology, vol. 34, no. 4, article 61, 2017.

[5] R. Lai, M. K. Rosenblum, and L. M. DeAngelis, "Primary CNS lymphoma: a whole-brain disease?" Neurology, vol. 59, no. 10, pp. 1557-1562, 2002.

[6] R. V. Jones, "Primary central nervous system lymphoma: radiologic-pathologic correlation," Radiographics, vol. 17, pp. 1497-1526, 1997.

[7] B. Bataille, V. Delwail, E. Menet et al., "Primary intracerebral malignant lymphoma: report of 248 cases," Journal of Neurosurgery, vol. 92, no. 2, pp. 261-266, 2000. 
[8] L. Fischer, P. Martus, M. Weller et al., "Meningeal dissemination in primary CNS lymphoma: Prospective evaluation of 282 patients," Neurology, vol. 71, no. 14, pp. 1102-1108, 2008.

[9] C.-C. Chan, J. L. Rubenstein, S. E. Coupland et al., "Primary vitreoretinal lymphoma: a report from an international primary central nervous system lymphoma collaborative group symposium," The Oncologist, vol. 16, no. 11, pp. 1589-1599, 2011.

[10] M. D. M. Jiménez de la Peña, L. G. Vicente, R. C. Alonso, S. F. Cabero, A. M. Suárez, and V. M. de Vega, “The Multiple Faces of Nervous System Lymphoma. Atypical Magnetic Resonance Imaging Features and Contribution of the Advanced Imaging," Current Problems in Diagnostic Radiology, vol. 46, no. 2, pp. 136145, 2017.

[11] U. Bühring, U. Herrlinger, T. Krings, R. Thiex, M. Weller, and W. Küker, "MRI features of primary central nervous system lymphomas at presentation," Neurology, vol. 57, no. 3, pp. 393396, 2001.

[12] W. Küker, T. Nägele, A. Korfel et al., "Primary central nervous system lymphomas (PCNSL): MRI features at presentation in 100 patients," Journal of Neuro-Oncology, vol. 72, no. 2, pp. 169$177,2005$.

[13] T. Sutherland, K. Yap, E. Liew, C. Tartaglia, M. Pang, and N. Trost, "Primary central nervous system lymphoma in immunocompetent patients: a retrospective review of MRI features," Journal of Medical Imaging and Radiation Oncology, vol. 56, no. 3, pp. 295-301, 2012.

[14] L. B. Patrick and N. A. Mohile, "Advances in primary central nervous system lymphoma," Current Oncology Reports, vol. 17, no. 12, article 60, 2015.

[15] H. Malikova, E. Koubska, J. Weichet et al., "Can morphological MRI differentiate between primary central nervous system lymphoma and glioblastoma?" Cancer Imaging, vol. 16, no. 1, p. 40, 2016.

[16] A. Mansour, M. Qandeel, H. Abdel-Razeq, and H. A. Abu Ali, "MR imaging features of intracranial primary CNS lymphoma in immune competent patients," Cancer Imaging, vol. 14, no. 1, article 22, 2014.

[17] A. C. Guo, T. J. Cummings, R. C. Dash, and J. M. Provenzale, "Lymphomas and high-grade astrocytomas: comparison of water diffusibility and histologic characteristics," Radiology, vol. 224, no. 1, pp. 177-183, 2002.

[18] F. Yamasaki, K. Kurisu, K. Satoh et al., "Apparent diffusion coefficient of human brain tumors at MR imaging," Radiology, vol. 235, no. 3, pp. 985-991, 2005.

[19] C.-H. Toh, M. Castillo, A. M.-C. Wong et al., "Primary cerebral lymphoma and glioblastoma multiforme: differences in diffusion characteristics evaluated with diffusion tensor imaging," American Journal of Neuroradiology, vol. 29, no. 3, pp. 471-475, 2008.

[20] S. J. Ahn, H. J. Shin, J.-H. Chang, and S.-K. Lee, "Differentiation between primary cerebral lymphoma and glioblastoma using the apparent diffusion coefficient: comparison of three different ROI methods," PLoS ONE, vol. 9, no. 11, Article ID e112948, 2014.

[21] M. Horger, M. Fenchel, T. Nägele et al., "Water diffusivity: Comparison of primary CNS lymphoma and astrocytic tumor infiltrating the corpus callosum," American Journal of Roentgenology, vol. 193, no. 5, pp. 1384-1387, 2009.

[22] K. Yamashita, T. Yoshiura, A. Hiwatashi et al., "Differentiating primary CNS lymphoma from glioblastoma multiforme: assessment using arterial spin labeling, diffusion-weighted imaging, and ${ }^{18}$ F-fluorodeoxyglucose positron emission tomography," Neuroradiology, vol. 55, no. 2, pp. 135-143, 2013.
[23] A. Doskaliyev, F. Yamasaki, M. Ohtaki et al., "Lymphomas and glioblastomas: Differences in the apparent diffusion coefficient evaluated with high b-value diffusion-weighted magnetic resonance imaging at 3 T,' European Journal of Radiology, vol. 81, no. 2, pp. 339-344, 2012.

[24] P. Kickingereder, B. Wiestler, F. Sahm et al., "Primary central nervous system lymphoma and atypical glioblastoma: multiparametric differentiation by using diffusion-, perfusion-, and susceptibility-weighted MR imaging," Radiology, vol. 272, no. 3, pp. 843-850, 2014.

[25] C. Calli, O. Kitis, N. Yunten, T. Yurtseven, S. Islekel, and T. Akalin, "Perfusion and diffusion MR imaging in enhancing malignant cerebral tumors," European Journal of Radiology, vol. 58, no. 3, pp. 394-403, 2006.

[26] J.-B. Wen, W.-Y. Huang, W.-X. Xu, G. Wu, D.-Y. Geng, and B. Yin, "Differentiating primary central nervous system lymphomas from glioblastomas and inflammatory demyelinating pseudotumor using relative minimum apparent diffusion coefficients," Journal of Computer Assisted Tomography, 2017.

[27] Y. S. Choi, H.-J. Lee, S. S. Ahn et al., "Primary central nervous system lymphoma and atypical glioblastoma: differentiation using the initial area under the curve derived from dynamic contrast-enhanced MR and the apparent diffusion coefficient," European Radiology, vol. 27, no. 4, pp. 1344-1351, 2017.

[28] J. Zhao, Z.-Y. Yang, B.-N. Luo, J.-Y. Yang, and J.-P. Chu, "Quantitative evaluation of diffusion and dynamic contrastenhanced MR in tumor parenchyma and Peritumoral area for distinction of brain tumors," PLoS ONE, vol. 10, no. 9, Article ID e0138573, 2015.

[29] I. S. Haldorsen, A. Espeland, and E.-M. Larsson, "Central nervous system lymphoma: characteristic findings on traditional and advanced imaging," American Journal of Neuroradiology, vol. 32, no. 6, pp. 984-992, 2011.

[30] S. S. Lu, S. J. Kim, N. Kim, H. S. Kim, C. G. Choi, and Y. M. Lim, "Histogram analysis of apparent diffusion coefficient maps for differentiating primary CNS lymphomas from tumefactive demyelinating lesions," American Journal of Roentgenology, vol. 204, no. 4, pp. 827-834, 2015.

[31] I. Harting, M. Hartmann, G. Jost et al., "Differentiating primary central nervous system lymphoma from glioma in humans using localised proton magnetic resonance spectroscopy," Neuroscience Letters, vol. 342, no. 3, pp. 163-166, 2003.

[32] Z.-C. Ye, J. D. Rothstein, and H. Sontheimer, "Compromised glutamate transport in human glioma cells: reductionmislocalization of sodium-dependent glutamate transporters and enhanced activity of cystine-glutamate exchange," The Journal of Neuroscience, vol. 19, no. 24, pp. 10767-10777, 1999.

[33] H. Aburano, F. Ueda, Y. Yoshie et al., "Differences between glioblastomas and primary central nervous system lymphomas in 1H-magnetic resonance spectroscopy," Japanese Journal of Radiology, vol. 33, no. 7, pp. 392-403, 2015.

[34] S. Jiang, H. Yu, X. Wang et al., "Molecular MRI differentiation between primary central nervous system lymphomas and highgrade gliomas using endogenous protein-based amide proton transfer MR imaging at 3 Tesla," European Radiology, vol. 26, no. 1, pp. 64-71, 2016.

[35] F. Roser, M. Saini, R. Meliss, H. Ostertag, M. Samii, and M. Bellinzona, "Apoptosis, vascularity, and proliferation in primary central nervous system lymphomas (PCNSL): a histopathological study," World Neurosurgery, vol. 62, no. 5, pp. 393-399, 2004. 
[36] I. H. Lee, S. T. Kim, H.-J. Kim, K. H. Kim, P. Jeon, and H. S. Byun, "Analysis of perfusion weighted image of CNS lymphoma," European Journal of Radiology, vol. 76, no. 1, pp. 48-51, 2010.

[37] S. Nakajima, T. Okada, A. Yamamoto et al., "Primary central nervous system lymphoma and glioblastoma: Differentiation using dynamic susceptibility-contrast perfusion-weighted imaging, diffusion-weighted imaging, and 18F-fluorodeoxyglucose positron emission tomography," Clinical Imaging, vol. 39, no. 3, pp. 390-395, 2015.

[38] S. Blasel, A. Jurcoane, O. Bähr, L. Weise, P. N. Harter, and E. Hattingen, "MR perfusion in and around the contrastenhancement of primary CNS lymphomas," Journal of NeuroOncology, vol. 114, no. 1, pp. 127-134, 2013.

[39] Z. Xing, R. X. You, J. Li, Y. Liu, and D. R. Cao, "Differentiation of primary central nervous system lymphomas from high-grade gliomas by rCBV and percentage of signal intensity recovery derived from dynamic susceptibility-weighted contrastenhanced perfusion MR imaging," Clinical Neuroradiology, vol. 24, no. 4, pp. 329-336, 2014.

[40] C. F. Lucchinetti, R. H. Gavrilova, I. Metz et al., "Clinical and radiographic spectrum of pathologically confirmed tumefactive multiple sclerosis," Brain, vol. 131, no. 7, pp. 1759-1775, 2008.

[41] C. A. Given II, B. S. Stevens, and C. Lee, "The MRI appearance of tumefactive demyelinating lesions," American Journal of Roentgenology, vol. 182, no. 1, pp. 195-199, 2004.

[42] A. L. Tievsky, T. Ptak, and J. Farkas, "Investigation of apparent diffusion coefficient and diffusion tensor anisotropy in acute and chronic multiple sclerosis lesions," American Journal of Neuroradiology, vol. 20, no. 8, pp. 1491-1499, 1999.

[43] H. S. Malhotra, K. K. Jain, A. Agarwal et al., "Characterization of tumefactive demyelinating lesions using MR imaging and invivo proton MR spectroscopy," Multiple Sclerosis Journal, vol. 15, no. 2, pp. 193-203, 2009.

[44] M. C. Mabray, B. A. Cohen, J. E. Villanueva-Meyer et al., "Performance of apparent diffusion coefficient values and conventional MRI features in differentiating tumefactive demyelinating lesions from primary brain neoplasms," American Journal of Roentgenology, vol. 205, no. 5, pp. 1075-1085, 2015.

[45] A. Rovira, I. Pericot, J. Alonso et al., "Serial diffusion-weighted MR imaging and proton MR spectroscopy of acute large demyelinating brain lesions: case report," American Journal of Neuroradiology, vol. 23, no. 6, pp. 989-994, 2002.

[46] J. J. Raizer, J. A. Koutcher, L. E. Abrey et al., "Proton magnetic resonance spectroscopy in immunocompetent patients with primary central nervous system lymphoma," Journal of NeuroOncology, vol. 71, no. 2, pp. 173-180, 2005.

[47] C. Enzinger, S. Strasser-Fuchs, S. Ropele, P. Kapeller, R. Kleinert, and F. Fazekas, "Tumefactive demyelinating lesions: conventional and advanced magnetic resonance imaging," Multiple Sclerosis Journal, vol. 11, no. 2, pp. 135-139, 2016.

[48] M. D. Kvarta, D. Sharma, R. J. Castellani et al., "Demyelination as a harbinger of lymphoma: a case report and review of primary central nervous system lymphoma preceded by multifocal sentinel demyelination," BMC Neurology, vol. 16, no. 1, article $72,2016$.

[49] L. Husseini, A. Saleh, G. Reifenberger, H.-P. Hartung, and B. C. Kieseier, "Inflammatory demyelinating brain lesions heralding primary CNS lymphoma," The Canadian Journal of Neurological Sciences, vol. 39, no. 1, pp. 6-10, 2012.

[50] T. Kuhlmann, A. Schröter, P. Dechent et al., "Diagnosis of a multifocal B cell lymphoma with preceding demyelinating central nervous system lesions by single voxel proton MR spectroscopy," Journal of Neurology, Neurosurgery \& Psychiatry, vol. 70, no. 2, pp. 259-262, 2001.

[51] S. Ng, H. Butzkueven, R. Kalnins, and C. Rowe, "Prolonged interval between sentinel pseudotumoral demyelination and development of primary CNS lymphoma," Journal of Clinical Neuroscience, vol. 14, no. 11, pp. 1126-1129, 2007.

[52] K. Brecher, F. H. Hochberg, D. N. Louis, S. de la Monte, and P. Riskind, "Case report of unusual leukoencephalopathy preceding primary CNS lymphoma," Journal of Neurology, Neurosurgery \& Psychiatry, vol. 65, no. 6, pp. 917-920, 1998.

[53] L. Alderson, M. R. Fetell, M. Sisti, F. Hochberg, M. Cohen, and D. N. Louis, "Sentinel lesions of primary CNS lymphoma," Journal of Neurology, Neurosurgery \& Psychiatry, vol. 60, no. 1, pp. 102-105, 1996.

[54] R. Bashir, M. Chamberlain, E. Ruby, and F. H. Hochberg, "T-cell infiltration of primary CNS lymphoma," Neurology, vol. 46, no. 2, pp. 440-444, 1996.

[55] W. Brück, A. Brunn, W. Klapper et al., "Differential diagnosis of lymphoid infiltrates in the central nervous system: Experience of the network lymphomas and lymphomatoid lesions in the nervous system," Der Pathologe, vol. 34, no. 3, pp. 186-197, 2013.

[56] R. Soffietti, A. Ducati, and R. Rudà, "Brain metastases," Handbook of Clinical Neurology, vol. 105, pp. 747-755, 2012.

[57] R. Soffietti, U. Abacioglu, B. Baumert et al., "Diagnosis and treatment of brain metastases from solid tumors: Guidelines from the European Association of neuro-oncology (EANO)," Neuro-Oncology, vol. 19, no. 2, pp. 162-174, 2017.

[58] S. F. Kralik, A. P. Kamer, and C. Y. Ho, "Diagnostic imaging of intracranial metastasis," Current Problems in Cancer, vol. 39, no. 2, pp. 99-112, 2015.

[59] W. H. Shim, H. S. Kim, C.-G. Choi, and S. J. Kim, "Comparison of apparent diffusion coefficient and intravoxel incoherent motion for differentiating among glioblastoma, metastasis, and lymphoma focusing on diffusion-related parameter," PLoS ONE, vol. 10, no. 7, Article ID e0134761, 2015.

[60] R. V. Gottumukkala, J. M. Romero, R. F. Riascos, R. Rojas, and R. S. Glikstein, "Imaging of the brain in patient," Topics in Magnetic Resonance Imaging, vol. 23, no. 5, pp. 275-291, 2014.

[61] P. C. Schroeder, M. J. D. Post, E. Oschatz, A. Stadler, J. Bruce-Gregorios, and M. M. Thurnher, "Analysis of the utility of diffusion-weighted MRI and apparent diffusion coefficient values in distinguishing central nervous system toxoplasmosis from lymphoma," Neuroradiology, vol. 48, no. 10, pp. 715-720, 2006.

[62] T. M. Ernst, L. Chang, M. D. Witt et al., "Cerebral toxoplasmosis and lymphoma in aids: perfusion MR imaging experience in 13 patients," Radiology, vol. 208, no. 3, pp. 663-669, 1998.

[63] W. Radwan, B. Lucke-Wold, I. A. Robadi, K. Gyure, T. Roberts, and S. Bhatia, "Neurosarcoidosis: Unusual presentations and considerations for diagnosis and management," Postgraduate Medical Journal, vol. 93, no. 1101, pp. 401-405, 2017.

[64] J. P. Zajicek, N. J. Scolding, O. Foster et al., "Central nervous system sarcoidosis-diagnosis and management," QJM: An International Journal of Medicine, vol. 92, no. 2, pp. 103-117, 1999.

[65] S. S. Rosenfeld, J. M. Hoffman, R. E. Coleman et al., "Studies of primary central nervous system lymphoma with fluorine18-fluorodeoxyglucose positron emission tomography," Indian Journal of Nuclear Medicine, vol. 33, no. 4, pp. 532-536, 1992.

[66] N. Kawai, K. Miyake, Y. Yamamoto, Y. Nishiyama, and T. Tamiya, " ${ }^{18}$ F-FDG PET in the diagnosis and treatment of 
primary central nervous system lymphoma," BioMed Research International, vol. 2013, Article ID 247152, 8 pages, 2013.

[67] S. Yamaguchi, K. Hirata, H. Kobayashi et al., "The diagnostic role of 18F-FDG PET for primary central nervous system lymphoma," Annals of Nuclear Medicine, vol. 28, no. 7, pp. 603609, 2014.

[68] N. A. Mohile, L. M. Deangelis, and L. E. Abrey, "The utility of body FDG PET in staging primary central nervous system lymphoma," Neuro-Oncology, vol. 10, no. 2, pp. 223-228, 2008.

[69] Y. Okada, T. Nihashi, M. Fujii et al., "Differentiation of newly diagnosed glioblastoma multiforme and intracranial diffuse large B-cell lymphoma using 11c-methionine and 18f-FDG PET," Clinical Nuclear Medicine, vol. 37, no. 9, pp. 843-849, 2012.

[70] Y. Kawase, Y. Yamamoto, R. Kameyama, N. Kawai, N. Kudomi, and Y. Nishiyama, "Comparison of 11C-methionine PET and 18F-FDG PET in patients with primary central nervous system lymphoma," Molecular Imaging and Biology, vol. 13, no. 6, pp. 1284-1289, 2011.

[71] N. Kosaka, T. Tsuchida, H. Uematsu, H. Kimura, H. Okazawa, and H. Itoh, "18F-FDG PET of common enhancing malignant brain tumors," American Journal of Roentgenology, vol. 190, no. 6, pp. W365-W369, 2008.

[72] K. Makino, T. Hirai, H. Nakamura et al., "Does adding FDGPET to MRI improve the differentiation between primary cerebral lymphoma and glioblastoma? Observer performance study," Annals of Nuclear Medicine, vol. 25, no. 6, pp. 432-438, 2011.

[73] Y. Zou, J. Tong, H. Leng, J. Jiang, M. Pan, and Z. Chen, "Diagnostic value of using 18F-FDG PET and PET/CT in immunocompetent patients with primary central nervous system lymphoma: A systematic review and meta-analysis," Oncotarget, vol. 8, no. 25, pp. 41518-41528, 2017.

[74] C. Schiepers, P. Van Hecke, R. Vandenberghe et al., "Positron emission tomography, magnetic resonance imaging and proton NMR spectroscopy of white matter in multiple sclerosis," Multiple Sclerosis Journal, vol. 3, no. 1, pp. 8-17, 1997.

[75] S. Takenaka, J. Shinoda, Y. Asano et al., "Metabolic assessment of monofocal acute inflammatory demyelination using MR spectroscopy and 11C-methionine-, 11C-choline-, and 18Ffluorodeoxyglucose-PET,' Brain Tumor Pathology, vol. 28, no. 3, pp. 229-238, 2011.

[76] M. V. Padma, M. Adineh, K. Pugar et al., "Functional imaging of a large demyelinating lesion," Journal of Clinical Neuroscience, vol. 12, no. 2, pp. 176-178, 2005.

[77] A. M. A. Maffione, L. Rampin, G. Grassetto, R. L'Erario, P. M. Colletti, and D. Rubello, "18F-FDG PET/CT in tumefactive multiple sclerosis," Clinical Nuclear Medicine, vol. 39, no. 8, pp. 750-751, 2014.

[78] F. Niccolini, P. Su, and M. Politis, "PET in multiple sclerosis," Clinical Nuclear Medicine, vol. 40, no. 1, pp. e46-e52, 2015.

[79] C. Chiavazza, A. Cistaro, P. Fania et al., "Reversible disconnection syndrome in a case of acute tumefactive demyelinating lesion: a PET study," Neurological Sciences, vol. 37, no. 12, pp. 2019-2023, 2016.

[80] Y. Ohe, T. Hayashi, K. Mishima et al., "Central nervous system lymphoma initially diagnosed as tumefactive multiple sclerosis after brain biopsy," Internal Medicine, vol. 52, no. 4, pp. 483-488, 2013.

[81] T. D. Westwood, C. Hogan, P. J. Julyan et al., "Utility of FDGPETCT and magnetic resonance spectroscopy in differentiating between cerebral lymphoma and non-malignant CNS lesions in
HIV-infected patients," European Journal of Radiology, vol. 82, no. 8, pp. e374-e379, 2013.

[82] M. Chan and E. Hsiao, "Neurosarcoidosis on FET and FDG PET/CT," Clinical Nuclear Medicine, vol. 42, no. 3, pp. 197-199, 2017.

[83] S. Canovi and D. Campioli, "Accuracy of flow cytometry and cytomorphology for the diagnosis of meningeal involvement in lymphoid neoplasms: a systematic review," Diagnostic Cytopathology, vol. 44, no. 10, pp. 841-856, 2016.

[84] R. Schroers, A. Baraniskin, C. Heute et al., "Diagnosis of leptomeningeal disease in diffuse large B-cell lymphomas of the central nervous system by flow cytometry and cytopathology," European Journal of Haematology, vol. 85, no. 6, pp. 520-528, 2010.

[85] D. Ekstein, D. Ben-Yehuda, E. Slyusarevsky, A. Lossos, E. Linetsky, and T. Siegal, "CSF analysis of IgH gene rearrangement in CNS lymphoma: relationship to the disease course," Journal of the Neurological Sciences, vol. 247, no. 1, pp. 39-46, 2006.

[86] T. Sasayama, S. Nakamizo, M. Nishihara et al., "Cerebrospinal fluid interleukin-10 is a potentially useful biomarker in immunocompetent primary central nervous system lymphoma (PCNSL)," Neuro-Oncology, vol. 14, no. 3, pp. 368-380, 2012.

[87] L. Nguyen-Them, M. Costopoulos, M.-L. Tanguy et al., "The CSF IL-10 concentration is an effective diagnostic marker in immunocompetent primary CNS lymphoma and a potential prognostic biomarker in treatment-responsive patients," European Journal of Cancer, vol. 61, pp. 69-76, 2016.

[88] Y. Song, W. Zhang, L. Zhang et al., "Cerebrospinal fluid IL10 and IL-10/IL-6 as accurate diagnostic biomarkers for primary central nervous system large B-cell lymphoma," Scientific Reports, vol. 6, no. 1, 2016.

[89] F. Strehlow, S. Bauer, P. Martus et al., "Osteopontin in cerebrospinal fluid as diagnostic biomarker for central nervous system lymphoma," Journal of Neuro-Oncology, vol. 129, no. 1, pp. 165-171, 2016.

[90] A. Viaccoz, F. Ducray, Y. Tholance et al., "CSF neopterin level as a diagnostic marker in primary central nervous system lymphoma," Neuro-Oncology, vol. 17, no. 11, pp. 1497-1503, 2015.

[91] D. D. Correa, L. Maron, H. Harder et al., "Cognitive functions in primary central nervous system lymphoma: literature review and assessment guidelines," Annals of Oncology, vol. 18, no. 7, pp. 1145-1151, 2007.

[92] M. B. Deutsch and M. F. Mendez, "Neurocognitive features distinguishing primary central nervous system lymphoma from other possible causes of rapidly progressive dementia," Cognitive and Behavioral Neurology, vol. 28, no. 1, pp. 1-10, 2015.

[93] Y. Zhang, Q. Zhang, X.-X. Wang, X.-F. Deng, and Y.-Z. Zhu, "Value of pretherapeutic DWI in evaluating prognosis and therapeutic effect in immunocompetent patients with primary central nervous system lymphoma given high-dose methotrexate-based chemotherapy: ADC-based assessment," Clinical Radiology, vol. 71, no. 10, pp. 1018-1029, 2016.

[94] R. F. Barajas Jr., J. L. Rubenstein, J. S. Chang, J. Hwang, and S. Cha, "Diffusion-weighted MR imaging derived apparent diffusion coefficient is predictive of clinical outcome in primary central nervous system lymphoma," American Journal of Neuroradiology, vol. 31, no. 1, pp. 60-66, 2010.

[95] M. J. Wieduwilt, F. Valles, S. Issa et al., "Immunochemotherapy with intensive consolidation for primary CNS lymphoma: a pilot study and prognostic assessment by diffusion-weighted mri," Clinical Cancer Research, vol. 18, no. 4, pp. 1146-1155, 2012. 
[96] F. E. Valles, C. L. Perez-Valles, S. Regalado, R. F. Barajas, J. L. Rubenstein, and S. Cha, "Combined diffusion and perfusion MR imaging as biomarkers of prognosis in immunocompetent patients with primary central nervous system lymphoma," American Journal of Neuroradiology, vol. 34, no. 1, pp. 35-40, 2013.

[97] S. R. Chung, Y. J. Choi, H. S. Kim, J. E. Park, W. H. Shim, and S. J. Kim, "Tumor vascular permeability pattern is associated with complete response in immunocompetent patients with newly diagnosed primary central nervous system lymphoma," Medicine, vol. 95, no. 6, Article ID e2624, 2016.

[98] N. Kawai, H.-N. Zhen, K. Miyake, Y. Yamamaoto, Y. Nishiyama, and T. Tamiya, "Prognostic value of pretreatment 18F-FDG PET in patients with primary central nervous system lymphoma: SUV-based assessment," Journal of Neuro-Oncology, vol. 100, no. 2, pp. 225-232, 2010.

[99] C. Muñiz, L. Martín-Martín, A. López et al., "Contribution of cerebrospinal fluid sCD19 levels to the detection of CNS lymphoma and its impact on disease outcome," Blood, vol. 123, no. 12, pp. 1864-1869, 2014.

[100] S. Roy, S. A. Josephson, J. Fridlyand et al., "Protein biomarker identification in the CSF of patients with CNS lymphoma," Journal of Clinical Oncology, vol. 26, no. 1, pp. 96-105, 2008.

[101] R. Schroers, A. Baraniskin, C. Heute et al., "Detection of free immunoglobulin light chains in cerebrospinal fluids of patients with central nervous system lymphomas," European Journal of Haematology, vol. 85, no. 3, pp. 236-242, 2010.

[102] J. L. Rubenstein, V. S. Wong, C. Kadoch et al., "CXCL13 plus interleukin 10 is highly specific for the diagnosis of CNS lymphoma," Blood, vol. 121, no. 23, pp. 4740-4748, 2013.

[103] A. Baraniskin, J. Kuhnhenn, U. Schlegel et al., "Identification of microRNAs in the cerebrospinal fluid as marker for primary diffuse large B-cell lymphoma of the central nervous system," Blood, vol. 117, no. 11, pp. 3140-3146, 2011.

[104] A. J. M. Ferreri, S. Dell'Oro, M. Foppoli et al., "MATILDE regimen followed by radiotherapy is an active strategy against primary CNS lymphomas," Neurology, vol. 66, no. 9, pp. 14351438, 2006.

[105] G. Citterio, M. Reni, and A. J. M. Ferreri, "Present and future treatment options for primary CNS lymphoma," Expert Opinion on Pharmacotherapy, vol. 16, no. 17, pp. 2569-2579, 2015.

[106] E. Thiel, A. Korfel, P. Martus et al., "High-dose methotrexate with or without whole brain radiotherapy for primary CNS lymphoma (G-PCNSL-SG-1): a phase 3, randomised, noninferiority trial," The Lancet Oncology, vol. 11, no. 11, pp. 10361047, 2010.

[107] M. N. Kerbauy, F. Y. Moraes, B. H. Lok et al., "Challenges and opportunities in primary CNS lymphoma: a systematic review," Radiotherapy \& Oncology, vol. 122, no. 3, pp. 352-361, 2017.

[108] A. J. M. Ferreri and G. Illerhaus, "The role of autologous stem cell transplantation in primary central nervous system lymphoma," Blood, vol. 127, no. 13, pp. 1642-1649, 2016.

[109] A. J. M. Ferreri, K. Cwynarski, E. Pulczynski et al., "Chemoimmunotherapy with methotrexate, cytarabine, thiotepa, and rituximab (MATRix regimen) in patients with primary CNS lymphoma: results of the first randomisation of the international extranodal lymphoma study group-32 (IELSG32) phase 2 trial," The Lancet Haematology, vol. 3, no. 5, pp. e217-e227, 2016.

[110] B. Kasenda, A. J. M. Ferreri, E. Marturano et al., "First-line treatment and outcome of elderly patients with primary central nervous system lymphoma (PCNSL)-a systematic review and individual patient data meta-analysis," Annals of Oncology, vol. 26, no. 7, pp. 1305-1313, 2015.

[111] K. Fritsch, B. Kasenda, E. Schorb et al., "High-dose methotrexate-based immuno-chemotherapy for elderly primary CNS lymphoma patients (PRIMAIN study)," Leukemia, vol. 31, no. 4, pp. 846-852, 2017.

[112] D. Kurzwelly, M. Glas, P. Roth et al., "Primary CNS lymphoma in the elderly: temozolomide therapy and MGMT status," Journal of Neuro-Oncology, vol. 97, no. 3, pp. 389-392, 2010.

[113] J. L. Rubenstein, E. Fraser, P. Formaker et al., "Phase I investigation of lenalidomide plus rituximab and outcomes of lenalidomide maintenance in recurrent CNS lymphoma," American Society of Clinical Oncology, Abstract 7502, 2016.

[114] C. Grommes, TJ. Kaley, C. Nolan et al., "Phase I study of single agent ibrutinib in recurrent/refractory primary (PCNSL) and secondary CNS lymphoma (SCNSL)," American Society of Clinical Oncology, Chicago, IL, USA, Abstract \#2046, 2016.

[115] E. Matsuki and A. Younes, "Checkpoint inhibitors and other immune therapies for hodgkin and non-hodgkin lymphoma," Current Treatment Options in Oncology, vol. 17, no. 6, article no. 31, 2016.

[116] Z. Gatalica, A. M. Vanderwalde, I. Rose et al., "Distribution of PD-L1 expression in diverse cancer types: experience with over 10, 000 cases," Journal of Clinical Oncology, vol. 34, abstract $11548,2016$.

[117] L. E. Abrey, T. T. Batchelor, A. J. Ferreri et al., "International primary CNS lymphoma collaborative group. Report of an international workshop to standardize baseline evaluation and response criteria for primary CNS lymphoma," Journal of Clinical Oncology, vol. 23, no. 22, pp. 5034-5043, 2005.

[118] G. Fossard, C. Ferlay, E. Nicolas-Virelizier et al., "Utility of posttherapy brain surveillance imaging in the detection of primary central nervous system lymphoma relapse," European Journal of Cancer, vol. 72, pp. 12-19, 2017.

[119] E. Tabouret, C. Houillier, N. Martin-Duverneuil et al., "Patterns of response and relapse in primary CNS lymphomas after first-line chemotherapy: imaging analysis of the ANOCEFGOELAMS prospective randomized trial," Neuro-Oncology, vol. 19, no. 3, pp. 422-429, 2017.

[120] H. Palmedo, H. Urbach, H. Bender et al., "FDG-PET in immunocompetent patients with primary central nervous system lymphoma: correlation with MRI and clinical follow-up," European Journal of Nuclear Medicine and Molecular Imaging, vol. 33, no. 2, pp. 164-168, 2006.

[121] J.-C. Jo, D. H. Yoon, S. Kim et al., "Interim 18F-FGD PET/CT may not predict the outcome in primary central nervous system lymphoma patients treated with sequential treatment with methotrexate and cytarabine," Annals of Hematology, vol. 96, no. 9, pp. 1509-1515, 2017.

[122] S. Mercadal, M. Cortés-Romera, P. Vélez, F. Climent, C. Gámez, and E. González-Barca, "Positron emission tomography combined with computed tomography in the initial evaluation and response assessment in primary central nervous system lymphoma," Medicina Clínica (English Edition), vol. 144, no. 11, pp. 503-506, 2015.

[123] S. Maza, R. Buchert, W. Brenner et al., "Brain and whole-body FDG-PET in diagnosis, treatment monitoring and long-term follow-up of primary CNS lymphoma," Radiology and Oncology, vol. 47, no. 2, pp. 103-110, 2013.

[124] S. J. Jang, K.-H. Lee, J. Y. Lee et al., "11C-methionine PET/CT and MRI of primary central nervous system diffuse large B-cell 
lymphoma before and after high-dose methotrexate," Clinical Nuclear Medicine, vol. 37, no. 10, pp. e241-e244, 2012.

[125] A. M. P. Omuro, L. S. Ben-Porat, K. S. Panageas et al., "Delayed neurotoxicity in primary central nervous system lymphoma," JAMA Neurology, vol. 62, no. 10, pp. 1595-1600, 2005.

[126] L. E. Abrey and D. D. Correa, "Treatment-related neurotoxicity," Hematology/Oncology Clinics of North America, vol. 19, no. 4, pp. 729-738, 2005.

[127] D. D. Correa, L. M. DeAngelis, W. Shi, H. Thaler, A. Glass, and L. E. Abrey, "Cognitive functions in survivors of primary central nervous system lymphoma," Neurology, vol. 62, no. 4, pp. 548$555,2004$.

[128] D. D. Correa, W. Shi, L. E. Abrey et al., "Cognitive functions in primary CNS lymphoma after single or combined modality regimens," Neuro-Oncology, vol. 14, no. 1, pp. 101-108, 2012.

[129] N. D. Doolittle, A. Korfel, M. A. Lubow et al., "Long-term cognitive function, neuroimaging, and quality of life in primary CNS lymphoma," Neurology, vol. 81, no. 1, pp. 84-92, 2013.

[130] K. Fliessbach, C. Helmstaedter, H. Urbach et al., "Neuropsychological outcome after chemotherapy for primary CNS lymphoma: a prospective study," Neurology, vol. 64, no. 7, pp. 11841188, 2005.

[131] E. A. Neuwelt, P. E. Guastadisegni, P. Várallyay et al., "Imaging changes and cognitive outcome in primary CNS lymphoma after enhanced chemotherapy delivery," American Journal of Neuroradiology, vol. 26, no. 2, pp. 258-265, 2005.

[132] R. Yamanaka, K. Morii, M. Sano et al., "Long-term survivors of primary central nervous system lymphoma," Japanese Journal of Clinical Oncology, vol. 47, no. 2, pp. 101-107, 2017.

[133] H. Harder, H. Holtel, J. E. C. Bromberg et al., "Cognitive status and quality of life after treatment for primary CNS lymphoma," Neurology, vol. 62, no. 4, pp. 544-547, 2004.

[134] I. T. Gavrilovic, A. Hormigo, J. Yahalom, L. M. DeAngelis, and L. E. Abrey, "Long-term follow-up of high-dose methotrexatebased therapy with and without whole brain irradiation for newly diagnosed primary CNS lymphoma," Journal of Clinical Oncology, vol. 24, no. 28, pp. 4570-4574, 2006.

[135] N. N. Laack and P. D. Brown, "Cognitive sequelae of brain radiation in adults," Seminars in Oncology, vol. 31, no. 5, pp. 702$713,2004$.

[136] T. Ichikawa, K. Kurozumi, H. Michiue et al., "Reduced neurotoxicity with combined treatment of high-dose methotrexate, cyclophosphamide, doxorubicin, vincristine and prednisolone (M-CHOP) and deferred radiotherapy for primary central nervous system lymphoma," Clinical Neurology and Neurosurgery, vol. 127, pp. 106-111, 2014. 


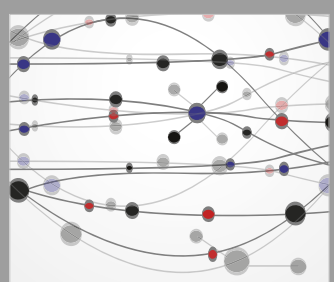

The Scientific World Journal
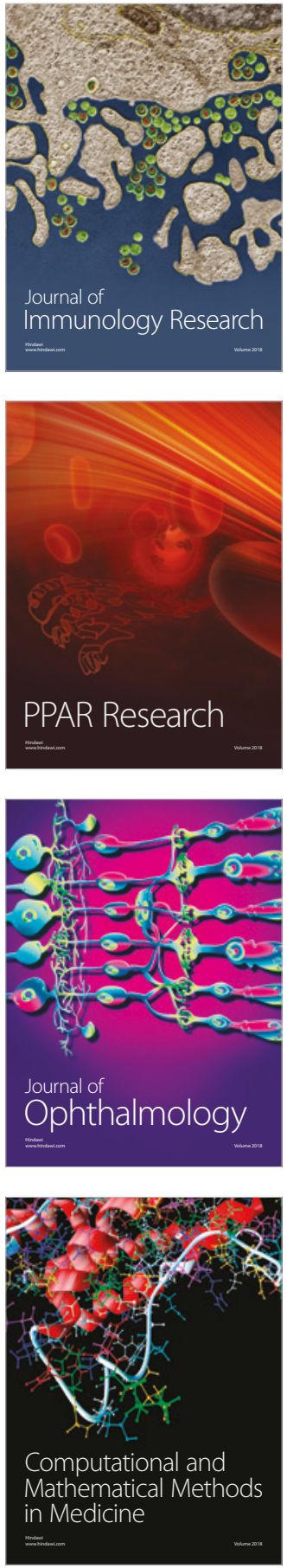

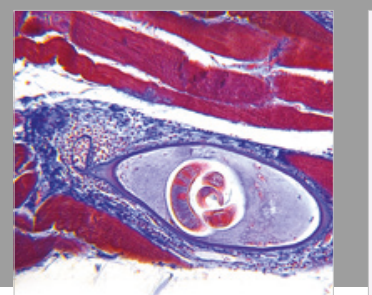

Gastroenterology Research and Practice

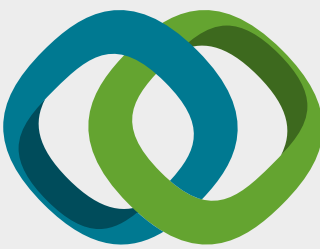

\section{Hindawi}

Submit your manuscripts at

www.hindawi.com
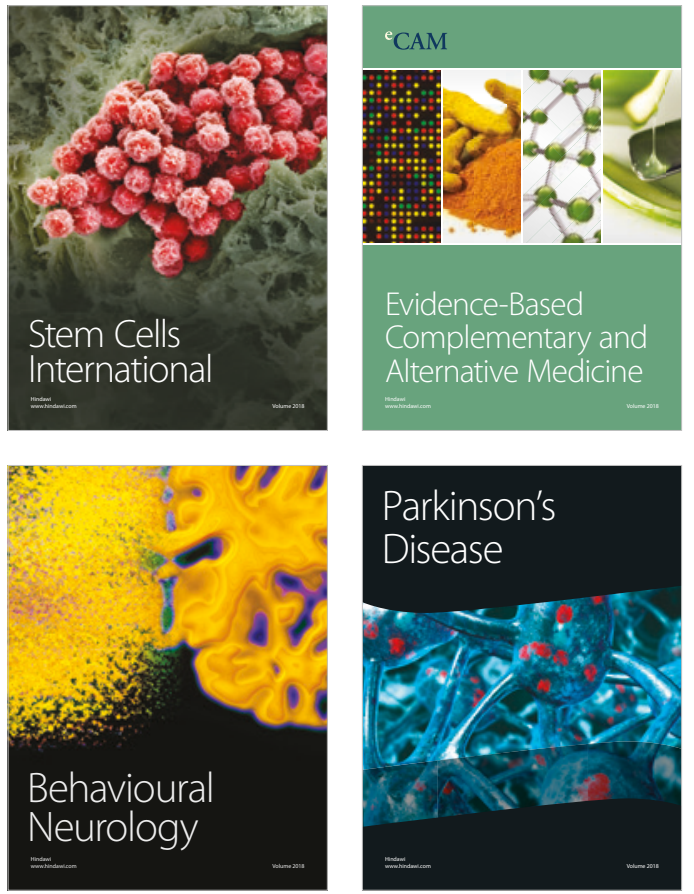

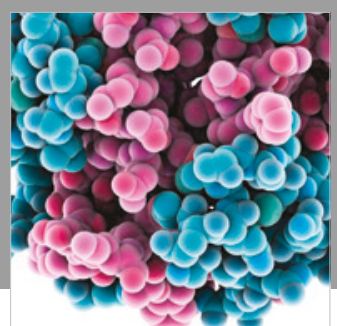

ournal of

Diabetes Research

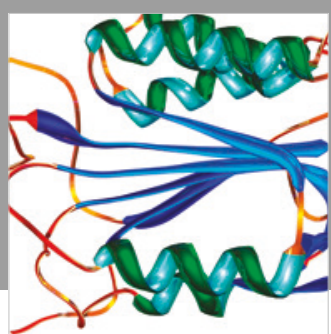

Disease Markers
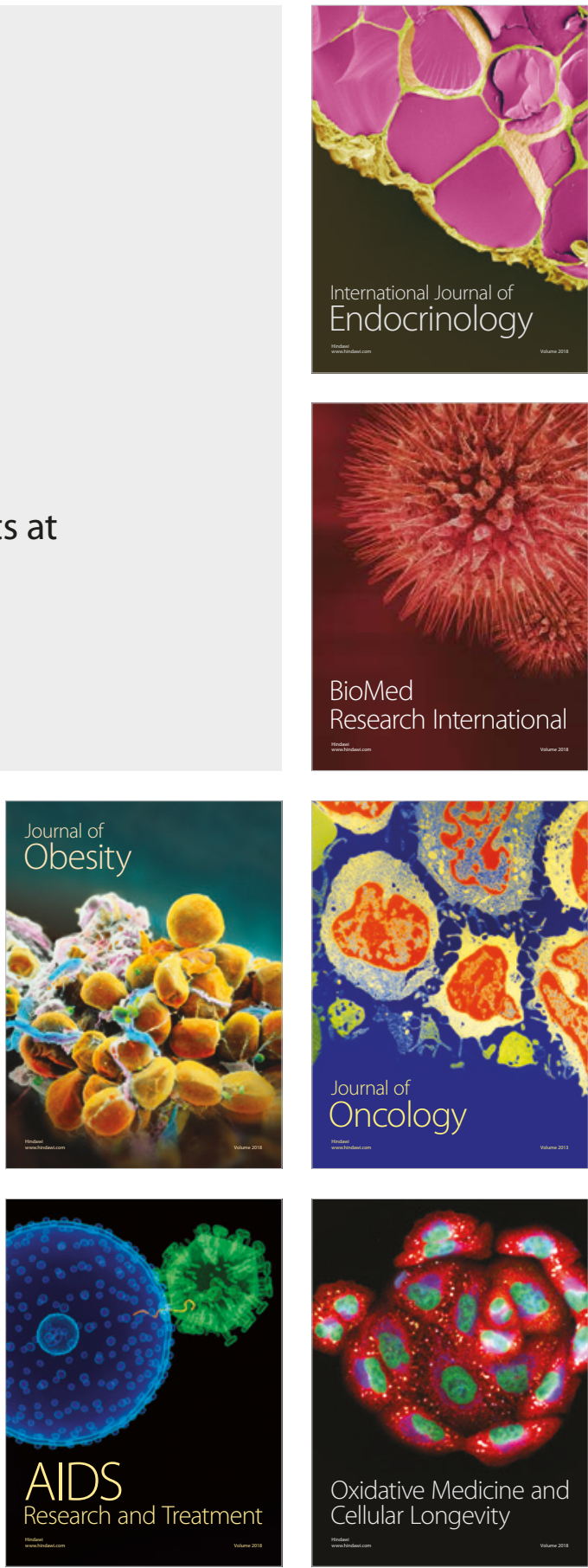